\title{
11 Die Zwischenreform 2013/2014
}

Angesichts vieler offenen Fragen im Wissenschaftsurheberrecht hatte die damalige Bundesregierung bei der Beschlussfassung des Zweiten Korbs zugesagt, unmittelbar mit den Planungen für einen Dritten Korb zu beginnen. Dieser Dritte Korb sollte nach den Vorstellungen auch der Wissenschaftspolitiker der Großen Koalition (CDU/CSU und SPD) explizit ein Wissenschaftskorb werden. ${ }^{352}$ Dieser „Dritte Korb“ mit dem umfassenden Anspruch eines wissenschaftsfreundlichen Urheberrechts ist allerdings nie realisiert worden. Die im Folgenden darzustellenden Reformen von 2014 können nicht als Ersatz für den Wissenschaftskorb angesehen werden.

Kurz vor Beendigung der Legislaturperiode (am 27.6.2013) wurde in einem Paket eine gesetzliche Regelung für die Nutzungsmöglichkeiten von verwaisten und vergriffenen Werken (vgl. Abschnitt 11.1) zusammen mit der Einfügung eines neuen Abschnitts 4 in $\S 38$ UrhG zur Regelung eines Zweitverwertungsrechts für Urheber (vgl. Abschnitt 11.3) im Bundestag verabschiedet. ${ }^{353}$ Dieses unter Zeitdruck verabschiedete Paket von 2013 kann höchstens als eine kleine Lösung angesehen werden, die aber auch nach der Reform von 2018 weiter gültig ist. Dazu merkte Ansgar Heveling (CDU/CSU - Teil der Regierungskoalition) bei der Beschlussfassung dieses Pakets an:

\begin{abstract}
Was ursprünglich ein ganzer „Korb“ an Maßnahmen zur Modernisierung des Urheberrechts werden sollte - es wäre der „Dritte Korb“ gewesen -, ist nun zu einem kleinen Bündel an Regelungen zusammengeschrumpft. Wir als CDU/CSU- Fraktion haben uns stets für eine umfassende Umsetzung des sogenannten Dritten Korbes und einer darin enthaltenen Anpassung des Urheberrechts an die Entwicklungen durch die Digitalisierung stark gemacht. ${ }^{354}$
\end{abstract}

Für sich genommen hätten beide neuen Normen Schritte in Richtung eines zeitgemäßen Urheberrechts sein können - zum einen durch Sicherung des Zugriffs auf

352 Federführend dafür war der Bundestagsausschuss für Bildung, Forschung und Technikfolgenabschätzung (Sprecher Jörg Tauss, MdB SPD), der anlässlich der abschließenden Beratung des Gesetzentwurfes der Bundesregierung für ein „Zweites Gesetz zur Änderung des Urheberrechtes in der Informationsgesellschaft” gefordert hatte „nun möglichst rasch [also in der neuen anstehenden Legislaturperiode, dann mit der CDU/CSU/FDP-Koalition] die Arbeiten an einem dritten Korb - einem Korb für die Belange von Bildung, Wissenschaft und Forschung in der Wissens-und Informationsgesellschaft - aufzunehmen.“ - so auch der Bundesrat nach der Empfehlung seiner Ausschüsse (Recht und Kultur) (Quelle: Pressemitteilung Aktionsbündnis 11/07 vom 21. September 2007 - https://bit.ly/2H5jfUO).

353 Der RefE für die UrhG-Reform 2003 - https://bit.ly/2CHn4gx; der RegE - https://bit.ly/ 2WbdwTH.

354 Haveling/CDU im Bundestag zur UrhG-Reform 2003 - https://bit.ly/2KVAHga. 
einen gewichtigen Teil des kulturellen Erbes für alle und zum anderen durch einen erweiterten Zugriff auf das überwiegend durch öffentliche Mittel produzierte und veröffentlichte Wissen. Aber um das Ergebnis der Darstellung in den folgenden Abschnitten vorwegzunehmen: Beurteilt man die Regelungen in der Zwischenreform unter dem Gesichtspunkt der Akzeptanz durch die davon Betroffenen bzw. unter der Praktikabilität und des Nutzens für die Allgemeinheit, so kann man nichts anders, als ihr Scheitern festzustellen - sieht man einmal von der speziellen Regelung für vergriffene Werke $a b$.

(1) Die angestrebte Massendigitalisierung von verwaisten Werken in den Beständen der Bibliotheken, Archiven und Mediatheken ist wegen zu rigider Vorgaben (Bedingung der sorgfältigen Suche, s. unten) durch das Gesetz ausgeblieben.

(2) Das Zweitverwertungsrecht, geplant als Verbesserung der Autonomie der Wissenschaftler und als freiere Nutzung publizierter Werke durch die Öffentlichkeit, wird - darauf deuten alle Hinweise hin - so gut wie gar nicht angenommen. Eine Mandatierung dieses Zweitverwertungsrechts bzw. die Einräumung eines institutionellen Zweitverwertungsrechts wurde nicht in Erwägung gezogen.

(3) Auch das Leistungsschutzrecht für Presseverleger kann - darüber ist sich die Fachwelt einig - nicht anders als ein Fehlschlag angesehen werden. ${ }^{355}$ Nichts hat sich in Erwartung von zusätzlichen Einnahmen für die Presseverleger geschweige denn für die betroffenen Journalisten verändert (ausführlicher in 11.4).

Alles kein Desaster - aber auch kein Muster wegweisender Urheberrechtspolitik. Auch bei der Zwischenreform bestätigt sich die These, dass es viel zu lange dauert, bis Regelungen verabschiedet werden, die dem Wissenschaftsurheberrecht zugeordnet sind. Die internationale Diskussion um verwaiste Werke (orphan works) begann in den USA schon 2005 und fand ihren Niederschlag 2006 in dem Report on Orphan Works des United States Copyright Office. ${ }^{356}$ Die Diskussion um das Zweitverwertungsrecht reichte sogar noch weiter zurück.

\subsection{Verwaiste Werke}

Am 1. Januar 2014 traten die neuen gesetzlichen Regelungen zur Nutzung von verwaisten Werken durch öffentliche Institutionen ( $\S \S 61$ bis 61c UrhG) in Deutschland

355 Tatsächlich war dieses Leistungsschutzrecht auch deshalb ein Fehlschlag, weil es vom EuGH im September 2019 für ungültig erklärt wurde, da die Bundesregierung es versäumt hatte, den Entwurf, wie erforderlich, zunächst der EU-Kommission vorzulegen - https://bit.ly/2v1658p. 356 United States Copyright Office (2006). „Report on Orphan Works“. Register of Copyrights. 
in Kraft. Der Weg dafür wurde durch eine EU-Richtlinie 2012 freigemacht. ${ }^{357}$ In der EU-Richtlinie von 2001 war eine solche Schranke nicht vorgesehen. Der dort vorgesehene Schrankenkanon verstand sich als abgeschlossen. Die EU hat dies dann 2012 mit der neuen Richtlinie geöffnet, so dass nun auch in Deutschland eine neue Schranke im Urheberrechtsgesetz eingefügt werden konnte.

Als „verwaist“ werden solche Werke bezeichnet, die entsprechend dem Datum ihrer Entstehung (bzw. 70 Jahre nach Tod des Urhebers) durch das Urheberrecht geschützt sind und für die die aktuellen Rechtsinhaber nicht bekannt sind bzw. nicht ausgemacht werden können. Das heißt aber nicht, dass durch den Verwaist-Status der Urheberrechtsschutz aufgegeben ist. In einer Situation rechtlicher Unsicherheit - was passiert, wenn doch ein Rechtsinhaber auftaucht und sein Recht reklamiert? - haben Bibliotheken gezögert, verwaiste Werke aus ihren Beständen zu digitalisieren und öffentlich über das Internet oder nur über das interne Netzwerk zugänglich zu machen. Ohne rechtliche Regelung müssten die Digitalisate bzw. aus ihnen entstandene Produkte wieder gelöscht oder sogar an auftauchende Rechtsinhaber Entschädigungen gezahlt werden.

Der Gesetzgeber hatte die Freiheit (und nach der Vorgabe der EU sogar die Pflicht), solche Unsicherheit durch Erweiterung des bestehenden Urheberrechts zu beseitigen, so dass die Nutzung verwaister Werke auch ohne Zustimmung der Rechtsinhaber erlaubt ist. Hier konfligierten zwei Rechtsinteressen: Einerseits besteht ein hohes öffentliches Interesse an der elektronischen Bereitstellung solcher Werke. Andererseits ist schon die Digitalisierung solcher Werke ein Eingriff in das Vervielfältigungsrecht als eines den Urhebern exklusiv zustehenden Verwertungsrechts. Entsprechend sind für das geltende Urheberrecht Verletzungen von Verwertungsrechten eine schwierig zu überwindende hohe Hürde. Das öffentliche Interesse wurde offensichtlich höher eingeschätzt als das Interesse unbekannter bzw. nicht ausgemachter Rechtsinhaber. Das war eine richtige und zeitgemäße Entscheidung. Aus informationsethischer Sicht besteht die Forderung, dass jedermann auf Werke Zugriff haben soll, die einmal öffentlich zugänglich gewesen waren. Jede Generation hat den Auftrag, das publizierte Wissen für Gegenwart und zukünftige Generationen verfügbar zu halten bzw. verfügbar zu machen. Ohne Rückgriff auf das kulturelle (auch verwaiste) Erbe ist, in Übereinstimmung mit der UNESCO-Konvention „Übereinkommen über den Schutz und die Förderung der Vielfalt kultureller Ausdrucksformen“358, Entwicklung nicht möglich. Zeitgemäß ist heute dieser Rückgriff nur in digitaler Form. Tatsächlich hatte der Gesetzgeber

357 Vorgabe EU-Richtlinie 2012/28/EU über bestimmte zulässige Formen der Nutzung verwaister Werke - https://bit.ly/2P3ZZhp; vgl. (Kuhlen 2010a) Freier Zugang zu den verwaisten Werken. 358 UNESCO-Konvention über den Schutz und die Förderung der Vielfalt kultureller Ausdrucksformen - https://bit.ly/2m6yaXJ. 
den Rückgriff auf das kulturelle Erbe als Begründung für die Regelung zu den verwaisten Werken verwendet. Deren Nutzung sei

nur zulässig, wenn die Institutionen zur Erfüllung ihrer im Gemeinwohl liegenden Aufgaben handeln, insbesondere wenn sie Bestandsinhalte bewahren und restaurieren und den Zugang zu ihren Sammlungen eröffnen, sofern dies kulturellen und bildungspolitischen Zwecken dient. (§61 Abs. 1)

Allerdings war der Respekt vor der bestehenden Urheberrechtssystematik mit dem übergroßen Respekt vor den Schöpfern von Werken so groß, dass solche Werke mit Verdacht auf „,verwaist“ nicht einfach genutzt werden können. Voraussetzung dafür ist, dass nur dann Werke als verwaist anerkannt und Nutzungen erlaubt werden, wenn ihnen eine sorgfältige Suche vorausgegangen ist und wenn diese zu keinem Erfolg geführt hatte. Die deutsche Regelung folgte dabei der US-amerikanischen Regelung und der durch die Richtlinie der EU: nur eine vorausgegangene sorgfältige (und im Ergebnis erfolglose, aber dennoch genau zu dokumentierende ${ }^{359}$ ) Suche (diligent search) nach dem/n Rechtsinhaber/n rechtfertige den Eingriff in die Rechte der Urheber bzw. der Rechte von verwertenden Verlagen. Von Seiten der deutschen Politik wurde entsprechend geltend gemacht, dass dem deutschen Gesetzgeber angesichts der 2012 erfolgten Vorgabe der EU-Richtlinie gar kein Handlungsspielraum für eine entsprechende deutsche Regelung gegeben war - vor allem bezüglich des Ausmaßes der Suche. Tatsächlich waren die Vorgaben der EU dafür sehr präzise und eröffneten möglicherweise in diesem Fall wenig kreativen Auslegungsspielraum.

Dennoch stellt sich auch hier die Frage (wie schon bei den Vorgaben aus der alten EU-Richtlinie von 2001), ob offensichtlich unbrauchbare Regelungsvorschläge zwangsläufig übernommen werden sollen/müssen. Der kompromisslose Anspruch auf sorgfältige Suche deutet darauf hin, dass die rechtsetzenden Akteure in Fällen wie diesen nur sehr bedingt fachliche Expertise über den Stand und die Möglichkeiten der für die Suche erforderlichen informationsmethodischen Instrumente eingeholt haben. Man schaue sich nur die Vorgaben aus dem An-

359 Eine kleine Anmerkung: Aus informationsmethodischer Sicht ist dieses Erfordernis der sorgfältigen Suche schon eine merkwürdige Konstruktion. Eine Suche wird in der Regel dann als Erfolg bezeichnet, wenn das Ergebnis der Suche für den Suchenden handlungsrelevante Information bereitstellt. Welche Motivation hat ein Suchender sorgfältig vorzugehen, wenn sein Ziel darin besteht, keine Information zu erhalten? Nur dann darf er handeln, also digitalisieren und das Digitalisat bereitstellen. Vielleicht war dieses gewisse Paradox der Grund dafür, die auf die Suche Verpflichteten nicht nur an ihr professionelles Suchethos zu erinnern, sondern sie auch auf eine ausführliche Dokumentation des Suchvorgangs zu verpflichten. Eine aufwändige Dokumentation eines negativen Ergebnisses ist nicht gerade motivierend. 
hang der EU-Richtlinie an (s. FN 357), die eingehalten werden müssen, wenn die Bedingung für eine sorgfältige Suche anerkannt werden soll. Zwar kann nach Art. 3 der EU- Richtlinie jedes Mitgliedsland bestimmen, welche Quellen für welche Kategorie (Druckwerke, multimediale Werke) als geeignet angesehen werden sollen, aber sie sollen „mindestens die im Anhang [der Richtlinie] aufgeführten relevanten Quellen“ einschließen. Das ist unrealistisch. Die deutsche Politik wird aber auch deshalb nicht von dem Vorwurf entlastet, ein folgenreiches Hindernis in das Gesetz übernommen zu haben, weil sie Zeit gehabt hatte, die Kritik an der Unbrauchbarkeit des „diligent search“ in die der EU-Richtlinie vorangegangenen Diskussion einzubringen. ${ }^{360}$ Diese Kritik gilt $u$. a. für die ungeklärten methodischen Aspekte der Automatisierung der Suche, auch für Auswahl und Verbindlichkeit der heranzuziehenden Ressourcen, für die zum Einsatz kommenden Suchalgorithmen, für die i.d.R. auch notwendige intellektuelle Suche in herkömmlichen Quellen, für die erforderliche Dokumentation bzw. Transparenz und Nachvollziehbarkeit der Suche, aber auch für die entstehenden Kosten durch die hohen Anforderung und die dafür aufzubauende Infrastruktur (Melderegister beim deutschen Patentamt und Datenbank bei der EU).

Als Ersatz für die sorgfältige Suche hätte sich eine Opt-out-Lösung angeboten: Es sollte eine zeitlich befristete (i. d. R. zwei Monate andauernde) öffentliche Bekanntmachung erfolgen, in der das Vorhaben der Digitalisierung und öffentlichen Bereitstellung solcher Werke angekündigt wird. Dafür käme die Deutsche Nationalbibliothek in Frage oder auch das Deutsche Patentamt (wie es jetzt bei den vergriffenen Werken der Fall ist). Wenn innerhalb dieser Frist kein Einspruch erfolgt, kann die Nutzung ohne weitere Lizenz durch eine Verwertungsgesellschaft erfolgen. Dieses Vorgehen sollte die entsprechenden Institutionen von möglichen Klagen bei später eventuell geltend gemachten Rechtsansprüchen freistellen. Um unbillige Rechteanmaßung zu verhindern, sollte derjenige, der die Rechte beansprucht, nachweisen, dass er diese auch tatsächlich hat. Im Sinne einer Opt-out-Lösung sollten die digitalisierten Objekte, zu denen ein Widerspruch eines Rechtsinhabers erfolgt, aus den Angebotsbeständen herausgenommen werden können. Eine nachträgliche Vergütung bei einer Nutzung ausschließlich im öffentlichen Interesse wäre (in Übereinstimmung mit dem EU-Entwurf) nicht angemessen.

Dieser Vorschlag wurde vor allem von den Mitgliedern im Rechtsausschuss nicht aufgegriffen - mit unguten Folgen: Die hohe, faktisch nicht zu überwindende Hürde der sorgfältigen Suche war dann, so stellte es sich bis heute heraus, der

360 Zumindest hätte sich die Bundesregierung spätestens 2017 in den Prozess der Formulierung von EU-DSM-RL dafür einsetzen können, dass die strikte Diligent-search-Vorgabe aufgegeben würde. Die EU hätte im Prinzip dafür offen sein können, da in die 2019 verabschiedeten RL eine neue Schranke für vergriffene Werke eingefügt wurde. 
entscheidende Grund dafür, dass von den Regelungen für verwaiste Werke zu wenig Gebrauch gemacht wurde. Über das Prinzip der „diligent search“ konnte es wegen des großen Aufwands nicht zu dem gewünschten Ergebnis der Massendigitalisierung der Bestände in den Bibliotheken und schon gar nicht in den Archiven und Mediatheken kommen. Tatsächlich zeigte sich seitdem, dass die Digitalisierung von verwaisten Werken wenig Fortschritt hatte machen können - wohingegen die (weniger restriktive) Regelung für die vergriffenen Werke durchaus erfolgreich von der Praxis aufgegriffen wurde (s. unten).

Dieser offensichtliche Fehlschlag der Nutzungsregelung für verwaiste Werke in Deutschland ist in hohem Maße bedauerlich. Man kann davon ausgehen, dass in deutschen Bibliotheken und Archiven vermutlich mehr als 30\% der Bibliotheksbzw. Medienarchivbestände zu den Kategorien der verwaisten und vergriffenen Werke zu zählen sind, die alle nur in analoger Form und überwiegend nur vor Ort eingesehen werden können. Bei einer Revision der Regelung für verwaiste Werke sollte vor allem das Kriterium der sorgfältigen Suche bedacht bzw. besser: aufgegeben werden. Für den Fehlschlag der Regulierung für verwaiste Werke war vermutlich auch die Entscheidung des Gesetzgebers verantwortlich, die kommerzielle Verwertung solcher Werke auszuschließen. ${ }^{361}$ In dieser Arbeit wurde oft kritisiert, dass Urheberrechtsregelungen i.d. R. zu stark auf die kommerzielle Verwertung abheben. Die Schranke über $§ 61$ ist ein Beispiel für das Gegenteil. Der starke Bezug auf das Gemeinwohlinteresse an verwaisten Werken war dem Gesetzgeber offenbar nicht verträglich mit der kommerziellen Verwertung verwaister Werke. Eine Erlaubnis zur Mitwirkung von kommerziellen Unternehmen bei der Massendigitalisierung von verwaisten Werken, ebenso die Einbeziehung von Organisationen und Initiativen aus dem genuin elektronischen Umfeld (z. B. Suchmaschinen oder Open-Content-Organisationen wie Wikipedia) in den Kreis der durch die Schranke Privilegierten wäre jedoch zielführend gewesen, da die öffentlich finanzierten Organisationen wie die durch $\S 61$ begünstigten die Mittel dafür kaum aufbringen können.

Die geringe Nutzung der neuen Schranke konnte offensichtlich auch nicht dadurch verhindert werden, dass auf europäischer Ebene durch das Amt der Euro-

361 Das Nutzungsrecht für verwaister Werke ist eine auf öffentliche Institutionen bezogene Schrankenregelung. Damit ist (unnötigerweise?) die auf nicht-kommerzielle Verwertung abzielende Nutzung von verwaisten Werken durch Einzelpersonen ausgeschlossen worden. Die hätte über die Privatkopierschranke (§53 Abs. 1) erlaubt sein können; nach dem BGH-Urteil vom 19.03.2014 (I ZR 35/13.) auch die Vervielfältigung (nicht die öffentliche Zugänglichmachung) unveröffentlichter Werke. Allerdings wäre in diesem Fall das Kriterium der diligent search noch schwieriger zu erfüllen. Das Kriterium der zeitlich befristeten öffentlichen Bekanntmachung des Nutzungsvorhabens wäre aber auch von Einzelpersonen zu erfüllen gewesen. 
päischen Union für Geistiges Eigentum (European Union Intellectual Property Office EUIPO)“ die Einrichtung und das Mangement „of a single publicly accessible online database on orphan works“362 betrieben wurde. Wenn im Sinne der EU-Richtlinie bzw. der neuen Schranke im deutschen Urheberrechtsgesetz ein Werk tatsächlich als verwaist bestimmt wurde, sollten von den durch die Richtlinie begünstigten Organisationen (also vor allem öffentliche Einrichtung wie Bibliotheken) Einträge in die EU-Datenbank entweder direkt vorgenommen werden oder (wie im deutschen Gesetz vorgesehen) an das Deutsche Patent- und Markenamt (PDMA) gemeldet werden, welches diese Meldungen dann an die EU-Datenbank weiterleitet. Ist ein Werk mit dem Status ,verwaist“ in die EU-Datenbank eingetragen, kann sich jede Einrichtung EU-weit darauf berufen; eine zusätzliche Suche nach den Rechtsinhabern ist dann nicht mehr erforderlich.

Die Datenbank erleichtert also nicht per se die sorgfältige Suche, macht aber eventuell, auch in grenzüberschreitender Sicht, Doppelarbeit unnötig. Die Datenbank steht auch den Urhebern/Rechtsinhabern zur Verfügung. Finden sie dabei ein Werk, für das sie Rechte reklamieren können, wird der Status als verwaistes Werk aufgehoben. Nach $\S 61$ b hat ,die nutzende Institution die Nutzungshandlungen unverzüglich zu unterlassen, sobald sie hiervon Kenntnis erlangt. Der Rechtsinhaber hat gegen die nutzende Institution Anspruch auf Zahlung einer angemessenen Vergütung für die erfolgte Nutzung." Wie gesagt - genutzt hat das offenbar wenig. Bei der Auswertung einer Umfrage des Intellectual Property Office der EU (EUIPO) von 2017 zur Nutzung dieser Datenbank konzentrierten sich die Antworten zur Frage nach Gründen, weshalb die EU-Online-Datenbank für verwaiste Werke nicht umfänglicher in Anspruch genommen wurde, besonders stark auf die Schwierigkeit, dem Kriterium der "diligent search" gerecht zu werden, ${ }^{363}$ z. B. schrieb ein Kommentator:

one respondent commented that there was a 'guilty until proven innocent' logic to the Orphan Works legislation that was obstructive to open access and sharing. The legislation was prohibitive and tended to scare people away. This respondent also put forward the suggestion that the use of the US-based principle of fair dealing in Europe would encourage more interest in recording orphan works. Moreover, there was no guidance on what constituted sufficient due diligence and no way to check if an orphan work had already been recorded somewhere else. (Referenz FN 362, p. 19)

362 EUIPO - https://bit.ly/2KKcHOo.

363 43,3\% very important; 16,4\% important; $9 \%$ slightly important; $11 \%$ fairly important und nur $6 \%$ not important. Fig. 14, p. 20. Ca. 60\% der Befragten sahen also die "diligent search" als größtes Hindernis für die Nutzung der Datenbank an. Vgl. auch Fig. 15, p. 21, wonach nur $10 \%$ der Benutzer zufrieden bzw. sehr zufrieden mit ihren Erfahrungen mit der diligent search waren (EUIPO-Report Referenz für alle Daten FN 362). 
Fazit. Durch $\S 61$ ist eine Schrankenregelung in das UrhG eingefügt worden, die eine institutionelle, nicht-kommerzielle Nutzung durch auf das Gemeinwohl ausgerichtet Kulturinstitutionen wie Bibliotheken erlaubt. Es ist sehr unglücklich, dass ein in hohem Maße politisch gewünschtes Ziel, den elektronischen Zugriff auf verwaiste Werke als Teil des kulturellen Erbes für jedermann zu erweitern, durch die unrealistische Bedingung der sorgfältigen Suche verfehlt wurde. Dass es anders geht, zeigt die Realisierung der vergriffenen Werke. Nicht, dass die dort gewählte Lizenzierungsprozedur hier als richtig oder sogar als wegweisend angesehen wird. Bei den vergriffenen Werken ist die Opt-out-Lösung mit einer Frist von 6 Wochen aber erlaubt, was der Schranke für die verwaisten Werke verweigert wurde. Das Beispiel der verwaisten Werke zeigt aber auch, dass zukunftsweisende und praxistaugliche Urheberrechtsregulierungen für Bildung und Wissenschaft nicht verabschiedet werden können, wenn an die alten (hier für dysfunktional gehaltenen) Fundamenten (vgl. Kap. 6) in der EU bzw. WIPO und entsprechend im deutschen UrhR festgehalten wird. In diesem Fall stand der Anspruch des individuellen Schöpfers von Werken im Wege.

\subsection{Vergriffene Werke}

Als ,vergriffen“ werden Werke bezeichnet, die zwar noch urheberrechtlich geschützt sind, aber regulär, also auf dem Verkaufsweg über den die Rechte besitzenden Verlag nicht mehr erwerbbar sind. Angebote über Antiquariate oder von Anbietern gebrauchter Bücher heben den Status als „vergriffen“ nicht auf. Auch verwaiste Werke sind vergriffene Werke. Aber die speziellen Regelungen für vergriffene Werke treffen für verwaiste Werke nicht zu, so dass der Regulierungserfolg für vergriffene Werke auf diese beschränkt bleibt. Für vergriffene Werke hatte die EU damals keine Richtlinienvorgabe gegeben. ${ }^{364}$ Deshalb war die Politik in Deutschland offenbar der Ansicht, dass keine neue (institutionelle) Schrankenregelung zugunsten von Organisationen wie Bibliotheken für vergriffene Werke möglich sei. Die Politik nutzte daher ihren Handlungsspielraum über eine Regelung durch das Urheberrechtswahrnehmungsgesetz (UrhWahrnG) ${ }^{365}$ bzw. über das diese Regelung ausführende Verwertungsgesellschaftengesetz. ${ }^{366}$

364 Das hat die EU 2019 in der neuen Urheberrechts-Richtlinie über Artikel 8-11 nachgeholt (s. unten).

365 UrhWahrnG - speziell über die neuen $\S \S 13 d$ (Vergriffene Werke) und 13e (Register vergriffener Werke) - https://bit.ly/323dijV. Vgl. (GRUR 2013) Stellungnahme zum RefE vom 20.2.2013.

366 Gesetz über die Wahrnehmung von Urheberrechten und verwandten Schutzrechten durch Verwertungsgesellschaften (Verwertungsgesellschaftengesetz - VGG), §51 (Vergriffene Werke) und $\S 52$ (Register vergriffener Werke) - https://bit.ly/2TlC3Xq. 
Das gesamte beschlossene Verfahren beruht auf dem Lizenzierungsansatz. Verwertungsgesellschaften (VG) werden durch das Gesetz berechtigt, an verwertende Organisationen wie Bibliotheken Lizenzen zu vergeben für die Vervielfältigung (durch Digitalisierung) und die öffentliche Zugänglichmachung vergriffener Werke, ohne dass ein Rechtsinhaber (Urheber, Verlag) die VG explizit mit der Wahrnehmung ihrer Rechte beauftragt hatte. Dafür gelten allerdings die in §51 VGG festgelegten Bedingungen, nämlich dass

(1) „es sich um vergriffene Werke handelt, die vor dem 1. Januar 1966 in Büchern, Fachzeitschriften, Zeitungen, Zeitschriften oder in anderen Schriften veröffentlicht wurden,

(2) sich die Werke im Bestand von öffentlich zugänglichen Bibliotheken, Bildungseinrichtungen, Museen, Archiven und von im Bereich des Film- oder Tonerbes tätigen Einrichtungen befinden,

(3) die Vervielfältigung und die öffentliche Zugänglichmachung nicht gewerblichen Zwecken dient,

(4) die Werke auf Antrag der Verwertungsgesellschaft in das Register vergriffener Werke (§13e) eingetragen worden sind und

(5) die Rechtsinhaber nicht innerhalb von sechs Wochen nach Bekanntmachung der Eintragung gegenüber dem Register ihren Widerspruch gegen die beabsichtigte Wahrnehmung ihrer Rechte durch die Verwertungsgesellschaft erklärt haben.“

Der Vorteil für eine Bibliothek besteht darin, dass sie durch die Lizenz von eventuell später auftretenden Rechtsansprüchen der Rechtsinhaber freigestellt wird. Der durch die neue Gesetzgebung sich eröffnende Spielraum wurde rasch genutzt. Die Grundlage für die Lösung für vergriffene Werke beruht auf einem 20.9.2011 abgeschlossenen „Memorandum of Understanding“ als eine gemeinsame Willenserklärung von Bibliotheken, Verlagen, Autoren und Verwertungsgesellschaften. ${ }^{367}$ Die Kultusministerkonferenz, in Vertretung des Bundes und der Länder, sowie die Verwertungsgesellschaften VG WORT und VG Bild-Kunst haben 2015 einen Rahmenvertrag zur Nutzung vergriffener Werke in Büchern vereinbart. ${ }^{368}$ Die Deutsche

$367 \mathrm{Ob}$ allerdings Autoren bzw. Autorenorganisationen an diesem Memorandum beteiligt wurden, konnte nicht mehr verifiziert werden. Keiner der mir bekannten zivilgesellschaftlichen Organisationen, die sich auch für Autorenrechte in Urheberrechtsangelegenheiten einsetzen, war an diesem Memorandum beteiligt. Kritisch zu diesem Memorandum und damit auch zu dem Lizenzierungsverfahren für vergriffene Werke (Ilja Braun 2011) Raus aus den Regalen. Braun schlug vor, „Autoren eine Infrastruktur zur Verfügung stellen, die es ihnen ermöglichen würde, Rechte an vergriffenen Werken, die derzeit nicht genutzt werden, in Eigenregie zu lizenzieren.“

368 Rahmenvertrag zur Nutzung vergriffener Werke in Büchern 2015 - https://bit.ly/2TRj30Z. 
Nationalbibliothek hatte dann zusammen mit dem DPMA einen Lieferservice „Vergriffene Werke“ aufgebaut, ${ }^{369}$ den durch das Gesetz berechtigten Organisationen (Bibliotheken etc.) nutzen konnten. Die Nutzung vergriffener Werke ist derzeit nur über diesen Lieferservice möglich. Die Nationalbibliothek reicht entsprechende Anträge der Nutzungsorganisationen über ein elektronisches Verfahren an die zuständige Verwertungsgesellschaft weiter (überwiegend VG WORT). Die Leistung der VG besteht zunächst darin, identifizierende Daten für die entsprechenden Werke, z. B. Titel, Urheber, Verlag etc., zu erheben und sie an das PDMA weiterzuleiten. Das PDMA ist, entsprechend §13e UrhWahrnG, für Einrichtung und Betrieb eines Registers vergriffener Werke zuständig. Die VG muss für jedes Werk einen Euro an das PDMA zahlen (die VG gibt dieses Kosten an die nutzenden Bibliotheken weiter). Dieser Betrag hat eher symbolische Bedeutung und deckt keineswegs die Einrichtungs- und laufenden Kosten des Patentamts. ${ }^{370}$

Die Register-Eintragungen werden auf der Internetseite des PDMA öffentlich zugänglich gemacht. Wenn sich innerhalb von 6 Wochen niemand mit Rechtsansprüchen meldet, können Lizenzverträge ausgestellt werden. Nach Ablauf der Offenlegungsfrist werden die Einrichtungen von der VG WORT über die erfolgreiche Lizenzierung benachrichtigt. ${ }^{371}$ Dafür müssen sie nach dem im Rahmenvertrag vorgesehenen Umfang für jedes Werk eine festgesetzte Gebühr an die Verwertungsgesellschaft entrichten. ${ }^{372}$ Nicht recht klar ist, wofür die VG WORT die Einnahmen

369 Lizenzierungsservice Vergriffene Werke der Deutschen Nationalbibliothek (DNB) - https: //bit.ly/2QQUz8n.

370 In der Begründung des Patentamts für die Gebühr heißt es: „Geht man von den voraussichtlichen Betriebskosten von ca. 72000 Euro pro Jahr aus, wäre bei zu erwartenden 10000 bis 20000 Anträgen pro Jahr eine Gebühr zwischen 3,60 und 7,20 Euro zu erheben, um die Betriebskosten zu decken. Diese Gebühr müsste noch deutlich höher angesetzt werden, würden außerdem die Einrichtungskosten von 430000 Euro berücksichtigt. Bei Eintragungsgebühren über 1 Euro ist jedoch wiederum zu befürchten, dass Gedächtniseinrichtungen die Regelungen zu vergriffenen Werken nicht in dem kulturpolitisch gewünschten Ausmaß in Anspruch nehmen und Verwertungsgesellschaften folglich deutlich weniger Anträge auf Eintrag beim DPMA stellen werden." Verordnung über das Register vergriffener Werke - https://bit.ly/2ZhLWc0.

371 VG WORT: Lizenz für Digitalisierung und öffentliche Zugänglichmachung vergriffener Werke https://bit.ly/2ZgjGST.

372 Im Rahmenvertrag wurde als angemessene Vergütung für Bücher die folgende Regelung vereinbart:

Für Bücher, die bis zum 31.12.1920 erschienen sind € 5; für Bücher, die zwischen 1.1.1921 und 31.1945 erschienen sind, $€ 10$ und für Bücher, die zwischen 1.1.1946 und 31.12.1965 erschienen sind $€ 15$ (dazu jeweils $7 \%$ Umsatzsteuer). Diese Beträge müssen von den die Lizenz wahrnehmenden Organisationen an die Verwertungsgesellschaft entrichtet werden, ebenso $1 €$ zur Erstattung des Betrags, den die Verwertungsgesellschaft für jeden Eintrag in das zentrale Register an das DPMA zahlen muss. 
von den Bibliotheken reklamiert bzw. verwendet werden. Ist es für die Ermittlung und Bereitstellung der bibliographischen, das Werk identifizierenden Daten? Oder werden Rückstände gebildet, um später bekanntwerdende Rechtsinhaber an den VG-Ausschüttungen zu beteiligen? Eine direkte Vergütung oder Entschädigung ist offenbar durch das Gesetz nicht vorgesehen. Jedermann kann Einsicht in das Register nehmen, so dass Urheber auf diese Weise „die gesetzliche Wahrnehmungsvermutung jederzeit widerlegen können“ (FN 369). Die entsprechende Zugänglichmachung durch Lizenznehmer wie Bibliotheken muss dann gelöscht werden. Allerdings sind diese nicht zu Entschädigungen verpflichtet.

Die EU hat mit ihrer 2019 beschlossenen Reform die vom EuGH geforderte Vorgabe nachgeholt und in „Art 18 Nutzung von vergriffenen Werken und sonstigen Schutzgegenständen durch Einrichtungen des Kulturerbes“ die schon 2013 beschlossene deutsche kollektive Lizenzierungsregelung für vergriffene Werke bestätigt:

Die Mitgliedstaaten legen fest, dass eine Verwertungsgesellschaft entsprechend den ihr von den Rechteinhabern erteilten Mandaten mit einer Einrichtung des Kulturerbes eine nicht ausschließliche Lizenzvereinbarung für nicht- kommerzielle Zwecke abschließen darf, die sich auf die Vervielfältigung, die Verbreitung, die öffentliche Wiedergabe oder die öffentliche Zugänglichmachung vergriffener Werke oder sonstiger Schutzgegenstände erstreckt, die sich dauerhaft in der Sammlung dieser Einrichtung befinden, unabhängig davon, ob alle Rechteinhaber, die unter die Lizenzvereinbarung fallen, der Verwertungsgesellschaft ein Mandat erteilt haben [...]. (Beginn von Art. 17 Abs. 1)

Die EU hat allerdings anerkannt, dass das kollektive Lizenzierungsverfahren nicht für alle Fälle vergriffener Werke passend ist, z. B.

wenn für eine bestimmte Art von Werken oder sonstigen Schutzgegenständen die kollektive Rechtewahrnehmung keine gängige Praxis ist oder wenn die maßgebliche Verwertungsgesellschaft für die Kategorie der Rechteinhaber und die jeweiligen Rechte nicht hinreichend repräsentativ ist.“ (EG 32). Daher sieht DSM-RL vor, dass es den Einrichtungen des Kulturerbes möglich ist, ,,vergriffene Werke oder sonstige Schutzgegenstände, die sich dauerhaft in ihrer Sammlung befinden, im Rahmen einer einheitlichen Ausnahme vom Urheberrecht und den verwandten Schutzrechten oder einer entsprechenden Beschränkung dieser Rechte in allen Mitgliedstaaten online zugänglich zu machen.

Das heißt, dass für bestimmte, überwiegend gar nicht für eine Veröffentlichung vorgesehene, aber kulturell bedeutsame Werke auch eine (rechtlich verbindliche) Schrankenregelung vorgesehen werden sollte. Es ist daher einer Überlegung wert, ob diese Erweiterung der Lösung für vergriffene Werke nicht weitergehend genutzt werden könnte. Fraglich, ob vielleicht effiziente, aber doch aufwändige und kostenintensives Lizenzierungsverfahren überhaupt das geeignete, das effektive Mittel sind, das gewünschte Ziel zu erreichen, nämlich vergriffene Werke als 
Teil des kulturellen Erbes der Öffentlichkeit über die nicht-gewerblichen Vermittlungs-/Gedächtnisorganisationen wie Bibliotheken frei zugänglich zu machen. ${ }^{373}$ Es stellt sich entsprechend die Frage, ob nicht durch eine rechtliche verbindliche Schrankenlösung das kulturpolitisch erwünschte Ziel des freien Zugangs zu einmal öffentlich gemachten, aber nun vergriffenen Werken für jedermann besser und auch kostengünstiger hätte erreicht werden können. Bei einer anfälligen Revision der deutschen Regelung für vergriffenen Werke sollte dies erwogen werden.

Vor allem aber sollte die in Deutschland restriktive zeitliche Vorgabe - nur Werke, die vor 1966 erschienen sind - durch eine offene Definition ersetzt werden, nämlich, wie aus bibliothekarischer Sicht gefordert, „dass das Werk in der jeweiligen oder in einer auf dem Markt konkurrierenden Form aktuell vom Rechtsinhaber nicht verwertet wird“.374 (de la Durantaye/Kuschel 2019) ${ }^{375}$ sehen sogar einen größeren Anpassungsbedarf durch den nun für die Mitgliedsländer verbindlich umzusetzenden Art. 8 von EU-DSM-RL2019:

Die Vorgaben der Richtlinie sind teils deutlich großzügiger als §§ VGG §51-VGG §52a VGG. Das betrifft sowohl die Werke als auch die erlaubten Nutzungsarten. Erfasst sind nicht nur Printwerke, sondern Werke und Schutzgegenstände aller Art. Sie dürfen vervielfältigt und öffentlich zugänglich gemacht, im Rahmen einer Lizenzierung nach Abs. 1 sogar öffentlich wiedergegeben und verbreitet werden. In anderen Bereichen sind die Vorgaben enger als nach deutschem Recht, etwa im Hinblick auf den Status eines Werkes als vergriffen. Es werden also einige Anpassungen des VGG und des UrhG erforderlich werden.

Fazit. Die befristete Auslegung war offensichtlich der entscheidende Faktor für den Erfolg der Regelung für vergriffene Werke. Was bei den verwaisten Werken offensichtlich nicht als zulässig angesehen wurde, wurde hier als sinnvoll und rechtmäßig eingeschätzt. Das jetzige Lizenzierungsverfahren beruht auf dem Opt-out-Ansatz: Erst einmal gilt das Verfahren für alle Rechtsinhaber für vergriffene Werke ohne deren explizite Zustimmung; sie können aber jederzeit die unterstellte Zustimmung widerrufen und damit aus dem Verfahren selbstbestimmt aussteigen.

373 Diese Diskussion ist von grundsätzlicher Bedeutung. Von Seiten der Verwertungsgesellschaften und der Verlagswirtschaft wird heute angesichts des „Erfolgs“ bei den vergriffenen Werken durch die jetzige Regelung in Deutschland stark dafür geworben, sämtliche Nutzungshandlungen über Lizenzierungsverfahren abzuwickeln. In 13.7 wird ausführlich auf die Frage Lizenzierung vs. Schrankenregelungen eingegangen.

374 Vgl. (Talke 2019) Warum die Urheberrechtsreform die Bibliotheken betrifft.

375 (de la Durantaye/Kuschel 2019) Vergriffene Werke größer gedacht. Vgl. (Peifer 2020) Anpassungsbedarf durch die neue Urheberrechtsrichtlinie. In der Tat hat das BMJV Mitte 2020 bei der Umsetzung von EU-DMS-RL einen Vorschlag vorgelegt, durch das der Begriff der ,vergriffenen Werke“ durch „nicht verfügbare Werke“ ersetzt werden soll. Das ermöglichte dann auch die Digitalisierung von Werken (wie z. B. in Nachlässen), die nie veröffentlicht worden sind. 
Anpassungsbedarf besteht durch die 2019 verabschiedete EU-DMS-RL. Hier gibt die Überlegung der EU, für bestimmte Werke auch eine Schrankenregelung anstelle der Lizenzierungslösung vorzusehen, vielleicht einen Anstoß, eine grundsätzlich andere rechtliche verbindliche Lösung für vergriffenen Werke $\mathrm{zu}$ finden - am attraktivsten durch eine direkte Verbindung der Regelungen für verwaiste und vergriffene Werke. Bei beiden ist das öffentliche Interesse an der Verfügbarkeit dieses kulturellen Erbes gleich groß. Dies sollte für Bildung und Wissenschaft freizügig verfügbar sein.

\subsection{Zweitverwertungsrecht}

Das Zweitverwertungsrecht wurde, mit Geltung 1.1.2014, durch die Einfügung eines Abs. 4 in $\S 38$ erweitert bzw. nach intensiven Diskussionen neu ausgerichtet, nachdem es im Prinzip schon 1965 über drei Absätze eingeführt war. ${ }^{376}$ Die früheren Absätze 1-3 von $\S 38$ bleiben mit einer Erweiterung um das Recht der öffentlichen Zugänglichmachung weiter gültig (s. unten). Hier der Text von §38 Abs. 4:

(4) Der Urheber eines wissenschaftlichen Beitrags, der im Rahmen einer mindestens zur Hälfte mit öffentlichen Mitteln geförderten Forschungstätigkeit entstanden und in einer periodisch mindestens zweimal jährlich erscheinenden Sammlung erschienen ist, hat auch dann, wenn er dem Verleger oder Herausgeber ein ausschließliches Nutzungsrecht eingeräumt hat, das Recht, den Beitrag nach Ablauf von zwölf Monaten seit der Erstveröffentlichung in der akzeptierten Manuskriptversion öffentlich zugänglich zu machen, soweit dies keinem gewerblichen Zweck dient. Die Quelle der Erstveröffentlichung ist anzugeben. Eine zum Nachteil des Urhebers abweichende Vereinbarung ist unwirksam.

Über $\S 38$ Abs. 4 wurde wie über kaum eine andere Norm - am ehesten vergleichbar mit § 52a-Alt - lange und vor allem kontrovers gestritten: ${ }^{377}$ Hier jeweils drei Beispiele für kritische Einwände gegen das Zweitverwertungsrecht und drei das Zweitverwertungsrecht unterstützende.

(1) Das Zweitverwertungsrecht, das ohnehin nur in Deutschland bzw. für die in Deutschland überwiegend lebenden Autoren gelte, könnte deutschen Autoren den Zugang zu internationalen Zeitschriften erschweren.

376 Eine ausführlich und sorgfältig referenzierte Dokumentation zur Entstehungsgeschichte und zum Gesetzgebungsverfahren, von den ersten Vorschlägen 2010 bis zur Verabschiedung 2013, findet sich auf der Website des Instituts für Urheber- und Medienrecht - https://bit.ly/2zejAR4. 377 Vgl. die durchaus kontroversen Stellungnahmen von Experten zum Zweitverwertungsrecht vor dem Rechtsausschuss des Bundestags am 10.6.2013 -https://bit.ly/2Hixp5k. 
(2) Das Zweitverwertungsrecht führe zur Entmündigung von Wissenschaftler und verletze deren Wissenschaftsfreiheit und führe zur Enteignung von Autoren und insbesondere der kommerziellen Verwerter.

(3) Das Zweitverwertungsrecht gefährde die Geschäftsmodelle auf den kommerziellen Informationsmärkten, die auf exklusiver Nutzung beruhten bzw. könne sogar zu einem Zusammenbruch der Publikumswirtschaft insgesamt führen.

(4) Das Zweitverwertungsrecht diene dem Schutz des geistigen Eigentums, sichere die Autonomie von Autoren und verstärke Wissenschaftsfreiheit.

(5) Das Zweitverwertungsrecht befördere das Interesse der Öffentlichkeit, nämlich zu dem mit öffentlichen Mitteln unterstützt produziertem Wissen freien Zugang zu erhalten, und das Interesse der Wirtschaft an „Folgeinnovationen“ aus neuem Wissen. ${ }^{378}$

(6) Das Zweitverwertungsrecht erweitere die Funktionsfähigkeit der wissenschaftlichen Fachkommunikation und steigere die individuelle Reputation der Autoren durch erhöhte Sichtbarkeit der publizierten Werke.

Das Zweitverwertungsrecht ist keine Schrankenregelung, sondern eine Regelung im Urhebervertragsrecht. Es ist - trotz der Benennung - kein Verwertungsrecht, ergänzt also nicht die anderen Verwertungsrechte in den $\S \S 15 \mathrm{ff}$. Trotzdem hat der Gesetzgeber in der Begründung von $\S 38$ Abs. 4 die Benennung „Zweitverwertungsrecht“ gewählt. Verwertungsrechte zielen aber nach herrschender Meinung überwiegend auf kommerzielle Verwertung ab. Das aber ist durch $\S 38$ Abs. 4 untersagt. ${ }^{379}$ Verschiedentlich wurde im Zusammenhang von $\S 38$ Abs. 4 von „Zweitveröffentlichungsrecht“ gesprochen - so von den Allianzorganisationen. ${ }^{380}$ Allerdings ist auch diese Bezeichnung nicht unproblematisch, da nach allgemeiner Einschätzung das Veröffentlichungsrecht mit der Erstveröffentlichung erloschen ist (entsprechend $\S 12$ Abs. 2 UrhG). Ein Zweitveröffentlichungsrecht kann es nach der Systematik des Urheberrechts nicht geben. „Zweitnutzungsrecht” wäre wohl die bessere Lösung

378 RegE Zweitverwertungsrecht; „Nur wenn Forschungsergebnisse frei verfügbar sind, können sie Grundlage weiterer Forschungsaktivitäten sein und die damit verbundenen positiven gesamtwirtschaftlichen Effekte auslösen. Forschung ist somit kein Selbstzweck. Wissen ist im globalen Wettbewerb ein entscheidender Faktor. Eine hohe Innovationskraft ist ohne ein produktives Wissenschaftssystem und einen effektiven Wissenstransfer nicht denkbar.“ - https://bit.ly/2NouagE. 379 Möglicherweise hat der Gesetzgeber also einen konstruktiven „Fehler“ gemacht, indem er bei seiner Terminologie an Verwerten=Nutzen gedacht hat. Dieser Ball kann zur Bestätigung der hier vertretenen Position aufgegriffen werden, dass „Verwerten“ in der Wissenschaft nicht kommerziell konnotiert ist, sondern auf Veröffentlichen abzielt (ausführlicher dazu in Abschnitt 5.5).

380 Vgl. (Allianz 2013) Eine Handreichung für die parlamentarischen Beratungen über ein unabdingbares Zweitveröffentlichungsrecht. 
gewesen, zumal dies in der Semantik auch mit § 12 Abs. 2 verträglich wäre; denn Zweitveröffentlichen kann man zwar nicht, aber das veröffentlichte Werke weiter nutzen, ist schon möglich. Auch die Einschränkung bei §38 Abs. 4, dass das Recht nicht für gewerbliche Zwecke genutzt werden darf, deutet darauf hin, dass es kein Verwertungsrecht ist, sondern eben nur ein Nutzungsrecht (vgl. Peukert, §38, RN 13). Wie auch immer - „Zweitverwertungsrecht“ ist nun einmal in der Sprache der Politik und in der Diskussion um $\S 38$ Abs. 4 üblich geworden. Daher wird hier diese (hier nicht auf eine kommerzielle Nutzung abzielende) Bezeichnung verwendet, auch wenn „Zweitnutzung“ gemeint ist.

\subsubsection{Zweitverwertungsrecht im Zusammenhang von $§ 38$ allgemein}

Was ist dieses Recht? Das Zweitverwertungsrecht ist das Recht von Autoren, über ihre Werke nach oder in Ergänzung zu einer kommerziellen Erstpublikation wieder (mehr oder weniger) frei verfügen zu können. ${ }^{381}$ Dieses nun neu bestimmte Autorenrecht soll dem Rechnung tragen, dass jeder Autor ein primäres Interesse daran hat, dass sein Werk so breit wie möglich wahrgenommen werden kann. Steinhauer (2010) bringt das im Titel eines kleinen Buchs zum Ausdruck: „Das Recht auf Sichtbarkeit“. Jeder Autor, so Steinhauer, hat dieses Recht als Bestandteil von Wissenschaftsfreiheit. Jede weitere Zugänglichmachung über die Erstveröffentlichung in einem kommerziellen Verlag hinaus erhöhe die Sichtbarkeit der Werke und die Reputation der Urheber. Das ist auch für Steinhauer die Grundlage für ein „verbindliches Zweitveröffentlichungsrecht” und zwar für jeden Wissenschaftler, ohne „Differenzierung zwischen öffentlich, privatwirtschaftlich oder selbst bezahlter Forschung“ (a. a. O., 47). ${ }^{382}$ Der Gesetzgeber hat das anders gesehen und die wissenschaftliche Berechtigtengruppe stark eingeschränkt (s. unten).

$381 \mathrm{Zu}$ unterscheiden ist das verbindliche Zweitveröffentlichungsrecht von der Praxis vieler, auch der großen Zeitschriftenverlage wie Elsevier, Wiley oder Springer, eine Selbstpublikation (self-archiving) der Werke ihrer Autoren als Postprint zu erlauben (also die mit der in der Zeitschrift inhaltlich übereinstimmenden, aber nicht verlagsformatierten Endversion des Artikels). Nach der Sherpa-Romeo-Liste (Stand 2/2020), welche über die Publikationspolitik vieler Verlage informiert, erlauben $81 \%$ der dort aufgeführten Verlage irgendeine Form von self-archiving - darunter gestatten 1064 Verlage Pre-print and Post-print archiving und 844 Verlage nur Post-print archiving (final draft post-refereeing) - https://bit.ly/2MumDx9. Wie gesagt „erlauben“ - dies ist kein einklagbares Recht. Es ist auch keineswegs gesichert, dass Self-archiving in jedem Fall mit den Prinzipien von Open Access übereinstimmt (entsprechend der Berliner Erklärung). Man muss sich die Publikationspolitik eines jeden Verlags gesondert anschauen.

382 (Steinhauer 2010) lehnt allerdings eine „Zweitveröffentlichungsverpflichtung“ grundsätzlich $\mathrm{ab}$, da diese Wissenschaftsfreiheit unzulässig einschränken würde. Wissenschaftsfreiheit als Grundrecht sei fundamental von der eigengesetzlichen Selbstbestimmung der Wissenschaft 
Ein Zweitverwertungsrecht ist kein Novum im UrhG. ${ }^{383}$ Es war auch bislang schon über $\S 38$ UrhG in drei Absätzen geregelt. Allerdings sah $\S 38$ Abs. 1 Satz 1 vor, dass „der Verleger oder Herausgeber im Zweifel [also auch, wenn keine ausdrückliche Vereinbarung vertraglich festgehalten wurde - RK] ein ausschließliches Nutzungsrecht zur Vervielfältigung, Verbreitung und öffentliche Zugänglichmachung“ erwirbt. ${ }^{384}$ Eine auch zeitlich uneingeschränkte vollständige Übertragung der Nutzungsrechte war aber 1965 dem Gesetzgeber doch zu weitgehend, und er sah in $\S 38$, Abs. 1-3 die folgenden Regelungen vor:

- $\$ 38$ Abs. 1, Satz 2 erlaubt dem Urheber, sein „Werk anderweit vervielfältigen, verbreiten und [nach der Reform von 2013 auch - RK] öffentlich zugänglich [zu] machen [...]“). Das gilt, wie oben erwähnt, auch für Abs. 2.

- Das Recht kann erst nach einer sogenannten Embargofrist von 12 Monaten wahrgenommen werden.

- Es gilt für Werke in einer periodisch erscheinenden Sammlung (sprich: Zeitschrift),

- aber auch für Werke in nicht-periodisch erscheinenden Sammlungen!

- Für Beiträge in Zeitungen erwirbt der Verlege nur ein einfaches Nutzungsrecht. Auch wenn der Autor ein ausschließliches Nutzungsrecht eingeräumt hat, darf er trotzdem seinen Artikel anderweitig vervielfältigen und verbreiten.

Das liest sich auf den ersten Blick wie ein Recht, das die Position der Urheber stark unterstützt. Auf den zweiten Blick erkennt man, dass so gut wie alle alten Erlaubnisse der Zweitverwertung durch die in den Absätzen 1-3-alt verbindliche Formulierung „wenn nichts anderes vereinbart ist“ außer Kraft gesetzt werden. Damit wurde auch in $\S 38$-alt dieses Zweitrecht faktisch ausgebremst, denn bis in die jüngste Vergangenheit war es die übliche Praxis, dass Autoren ihre Verwertungsrechte i.d. R. exklusiv als Nutzungsrechte an die Verlage/Verleger übertragen ohne Einschränkung und auch für alle, (ab 2008) auch für bis dahin unbekannte Anwendungen. Vor allem jüngere Wissenschaftler zu Beginn ihrer Karriere waren somit selten in der Lage, gegenüber den Verlagen ihr an sich durch $\S 38$ zugestandenes Zweitveröffentlichungsrecht durchzusetzen.

geprägt.“: „Wie künftig wissenschaftlich publiziert werden soll, kann nur sie [die Wissenschaft RK] selbst entscheiden“ (a. a. O. 48). Wir teilen diese Auffassung nicht, dass auch das Zweitveröffentlichungsrecht ein aus Wissenschaftsfreiheit abgeleitetes Recht sei.

383 Die folgende Darstellung folgt in weiten Teilen und Formulierungen den beiden Arbeiten (Kuhlen 2011): Der Streit um die Regelung des Zweitveröffentlichungsrechts im Urheberrecht und (Kuhlen 2014) Interdependenzen zwischen Informationsethik und politischem Handeln.

384 Die öffentliche Zugänglichmachung ist in Abs. 1 und analog auch in Abs. 2 erst mit der Reform von 2013/2014 aufgenommen worden. 
Das war auch für die Politik keine akzeptable Situation. Schon 2004, bei der Planung des Zweiten Korbs, fand das Zweitverwertungsrecht Eingang in die politische Auseinandersetzung. ${ }^{385}$ Konkreter Anlass war die Debatte um die dann auch erfolgte Abschaffung von Abs. 4 in $\S 31$ UrhG - Einräumung von Nutzungsrechten. Dieser Plan blieb nicht ohne Widerspruch. So wies die Deutsche Initiative für Netzwerkinformation (DINI e. V.) ${ }^{386}$ darauf hin, dass bei Wegfall des erwähnten Absatzes 4 ,Verleger versuchen, durch Retrodigitalisierung eine ähnlich verstärkte Verwertungsrechtsposition zu erreichen, wie dies bei original digitalen Publikationen der Fall ist (volles Lizenzierungsrecht ohne Schrankenregelungen). “ Dadurch drohe zum einen die Gefahr, dass Urheber Verwertungsrechte an die Verleger verlören, zum anderen, dass Bibliotheken nicht mehr aus ihren eigenen Beständen Werke digitalisieren und der Öffentlichkeit über eine „Zweitverwertung“ frei zugänglich machen dürfen. Auch Bibliotheken, so DINI, müsse im öffentlichen Interesse das Zweitverwertungsrecht über die Digitalisierung zugestanden werden. ${ }^{387}$ Den Bedenken wurde vom Gesetzgeber nicht Rechnung getragen (ausführlicher 10.2). Auch wurde 12 Jahre später bei der vollzogenen Reform für das Zweitverwertungsrecht der DINI-Forderung nach einem institutionellen Zweitverwertungsrecht nicht entsprochen (dazu ausführlich in 11.3.6).

Unterstützung für ein institutionelles Zweitverwertungsrecht kam ebenfalls 2004 durch einen Artikel von (Pflüger/Ertmann 2004). ${ }^{388}$ Darin wurde vorgeschlagen, dass in den für die Hochschulen zuständigen Landesgesetzen eine Zweitverwertungsverpflichtung der an den Hochschulen beamteten oder angestellten Wissenschaftler verankert werden sollte. Dieser Vorschlag ist dann allerdings zunächst nicht weiterverfolgt worden, nachdem Hansen $2005^{389}$ in seinem Vorschlag für ein Zweitverwertungsrecht diesen Plan als nicht gesetzeskompatibel zurückgewiesen hatte. ${ }^{390}$ Hansen machte in seinem Beitrag klar, dass ein Zweitver-

385 Die Darstellung in diesem Absatz folgt (Kuhlen 2014a) Interdependenzen zwischen Informationsethik und politischem Handeln.

386 Im November 2004 formulierte die DINI-Arbeitsgruppe „Urheberrecht“ umfassende Kritik an dem Referentenentwurf des BMJ vom 27.09.2004 für ein Zweites Gesetz zur Regelung des Urheberrechts in der Informationsgesellschaft ("2. Korb").

387 Ähnliche Argumentation in dem Rechtspolitischen Positionspapier des dbv zum Referentenentwurf für ein Zweites Gesetz zur Regelung des Urheberrechts in der Informationsgesellschaft vom 11. Oktober 2004.

388 (Pflüger/Ertmann 2004) E-Publishing und Open Access - Konsequenzen für das Urheberrecht im Hochschulbereich.

389 (Hansen 2005) Zugang zu wissenschaftlicher Information - alternative urheberrechtliche Ansätze, S. 386.

390 Pflüger als weiter in der Wissenschaftspolitik von Baden-Württemberg aktive Koautor des Artikels von 2004 hatte allerdings diese Idee eines institutionellen Zweitverwertungsrechts nie 
wertungsrecht nicht durch eine neue Schranke eingeführt werden könnte. Das wurde von ihm vor allem aus unionsrechtlichen Gründen verworfen. ${ }^{391} \mathrm{Er}$ machte daher den Vorschlag, das Zweitverwertungsrecht in das Urhebervertragsrecht einzubetten, und er betonte den „offeneren Zugang“ als Zweck der Regelung: „Schließlich bietet sich de lege ferenda eine Regelung im Urhebervertragsrecht als urheberrechtliches Instrument an, um öffentlich-rechtliche Regelungen zugunsten eines offeneren Zugangs zu Wissen zu flankieren." Der Bundesrat hatte dann 2007 den Vorschlag von Hansen aufgegriffen und eine entsprechende Neuregelung für $\S 38$ UrhG im Rahmen des Zweiten Korbs vorgeschlagen. ${ }^{392}$ Dieser Vorschlag wurde dann von der Bundesregierung abgelehnt ${ }^{393}$ - unter anderem mit dem (den Stand der Diskussion ignorierenden) Hinweis, dass damit eine neue Schrankenregelung geschaffen würde. Das aber sei entsprechend InfoSoc 2001 mit der abgeschlossenen Liste für Schranken nicht erlaubt. Das Zweitverwertungsrecht war 2007 offensichtlich noch nicht politisch gewollt. 2007 hätte es wegweisend sein können. 2014 kam es zu spät.

Die gesetzgebende Politik blendete sich also zunächst aus dieser Diskussion aus. Sie wurde dann aber mit einigen Initiativen/Petitionen aus der breiteren Öffentlichkeit konfrontiert, durch die das Zweitverwertungsrecht-Thema im Zusammenhang der freien öffentlichen Zugänglichmachung des mit öffentlichen Mitteln finanzierten Wissens gestellt wurde. Ob es der öffentliche Druck war, sei

gänzlich aufgegeben. Wir gehen darauf in Abschnitt 11.3.6 ein, als das Land Baden-Württemberg, wohl nicht zuletzt durch Pflügers Einwirken, nach Gültigwerden des Zweitverwertungsrechts 2014 versuchte, eine landesspezifische Lösung für ein solches institutionelle Zweitverwertungsrecht durchzusetzen.

391 Die Liste der Schrankenregelungen in der weiter verbindlichen InfoSoc Richtlinie 2001 wurde als abgeschlossen angesehen. Ein Zweitverwertungsrecht war dabei nicht vorgesehen.

392 Vorschlag des Bundesrats zur Regelung eines Zweitverwertungsrechts in seiner Stellungnahme zum Regierungsentwurf des Zweiten Korbes (BR-Drs. 257/06): „An wissenschaftlichen Beiträgen, die im Rahmen einer überwiegend mit öffentlichen Mitteln finanzierten Lehr- und Forschungstätigkeit entstanden sind und in Periodika erscheinen, hat der Urheber auch bei Einräumung eines ausschließlichen Nutzungsrechts das Recht, den Inhalt längstens nach Ablauf von sechs Monaten seit Erstveröffentlichung anderweitig öffentlich zugänglich zu machen, soweit dies zur Verfolgung nicht kommerzieller Zwecke gerechtfertigt ist und nicht in der Formatierung der Erstveröffentlichung erfolgt. Dieses Recht kann nicht abbedungen werden.“

393 Gegenäußerung der Bundesregierung zur Stellungnahme des Bundesrates zum Entwurf eines Zweiten Gesetzes zur Regelung des Urheberrechts in der Informationsgesellschaft (BR-Drs. 257/06 - Beschluss). Zumindest aber hatte bei der Verabschiedung des Zweiten Korbs der Ausschuss des Deutschen Bundestags für Bildung, Forschung und Technikfolgenabschätzung das Bundesjustizministerium (BMJ) aufgefordert, eine „Prüfung eines Zweitverwertungsrechts für Urheber von wissenschaftlichen Beiträgen, die überwiegend im Rahmen einer mit öffentlichen Mitteln finanzierten Lehr- und Forschungstätigkeit entstanden sind (§38 UrhG), vorzunehmen.“ 
dahingestellt - aber der verspätet eingeschlagene Weg zum Zweitverwertungsrecht wurde vor allem dadurch frei, dass sich in der Bundesregierung ein längst überfälliger Perspektivenwechsel durchsetzte, nämlich dass es sich bei dem Zweitverwertungsrecht um eine urhebervertragsrechtliche Lösung handele. Es gab also keinen dogmatischen Grund mehr, diese Reform nicht zu realisieren.

\subsubsection{Zu den Details/Beschränkungen des in $\S 38$ Abs. 4 realisierten Zweitverwertungsrechts}

Angesichts der auch für die damalige Bundesregierung offensichtlichen Machtasymmetrie durch das wirtschaftliche Ungleichgewicht zugunsten der Verleger sollten urhebervertragsrechtliche Regelungen die Urheber gegenüber den Verlegern stärken. Als entscheidend für die Reform wurde angesehen, die bislang in $\S 38$ vorgesehene Einschränkung „wenn nichts anderes vereinbart” durch eine verbindliche, unabdingbare Vorgabe „Eine zum Nachteil des Urhebers abweichende Vereinbarung ist unwirksam“ abzulösen. Das ist in der Tat die zentrale Entscheidung des Gesetzgebers. Kein Verleger kann seitdem einen Vertrag mehr abschließen, welcher das Zweitverwertungsrecht des Urhebers ausschließt. Tut er es trotzdem, so ist dieser Vertrag, entsprechend Satz 3 in $\S 38$ Abs. 4, unwirksam. Man sprich daher von einem zwingenden oder nicht abdingbaren Zeitverwertungsrecht.

„Zwingend“ bedeutet aber für den Gesetzgeber bislang nicht, dass dieses Recht von dem jeweiligen Urheber unbedingt wahrgenommen werden muss. Das wäre eine mandatorische Verpflichtung des Urhebers. $§ 38$ Abs. 4 ist also zunächst als Fortschritt gegenüber dem alten $\S 38 \mathrm{zu}$ werten, dessen Abs. 1-3 aber weitgehend unverändert erhalten geblieben sind. Allerdings bleibt der Gesetzgeber auch mit $\S 38$ Abs. 4, wie wir zeigen werden, auf halbem Wege stecken, so dass die anvisierten Ziele nur teilweise erreicht werden dürften, als da sind: Stärkung der Autonomie der Urheber, größere Sichtbarkeit der Werke und Nutzung der erarbeiteten Forschungsergebnisse durch die Öffentlichkeit, nicht zuletzt die Unterstützung der Innovationsfähigkeit der Wirtschaft. Die zentralen Regelungen für das Zweitverwertungsrecht durch $\S 38$ Abs. 4 werden wie folgt zusammengefasst: ${ }^{394}$

(1) Seit Beginn des Jahres 2014 ist es durch $\S 38$ Abs. 4 in Deutschland Autoren an bestimmten Institutionen erlaubt, nach der Erstveröffentlichung von Artikeln in wissenschaftlichen

394 Vgl. (Bruch/Pflüger 2014) Das Zweitveröffentlichungsrecht des § 38 Absatz 4 UrhG; (Kuhlen 2011a) Der Streit um die Regelung des Zweitveröffentlichungsrechts; (Kuhlen 2013b) Manifestierung der Dreiklassengesellschaft; (de la Durantaye 2014b) Stellungnahme zu dem Entwurf eines Gesetzes zur Nutzung verwaister und vergriffener Werke und einer weiteren Änderung des Urheberrechtsgesetzes. 
(bis dahin i. d. R. kommerziell vertriebenen) Fachzeitschriften diese über das Internet selbst öffentlich zugänglich zu machen.

(2) Die Zweitverwertung gilt nur für Werke in periodisch (mindestens zweimal pro Jahr) erscheinenden Sammlungen - gemeint sind also Zeitschriften.

(3) Das Zweitverwertungsrecht gilt nur für wissenschaftliche Arbeiten, die im Rahmen einer Forschungstätigkeit entstanden sind. Dazu können auch wissenschaftliche bildungsbezogene Arbeiten gerechnet werden, aber nicht rein auf Didaktik bezogene Texte. 395

(4) Die Zweitverwertung darf nicht gewerbliche Zwecke verfolgen.

(5) Die Zweitverwertung ist auf den Geltungsbereich des deutschen Rechts beschränkt.

(6) Bei Arbeiten mit Mehrfachautoren haben alle beteiligten Autoren das Zweitverwertungsrecht. Derjenige, der das Recht wahrnimmt, muss die Zustimmung der anderen Autoren einholen. Die Zustimmung kann nur aus zwingenden Gründen verweigert werden.

(7) Nicht eindeutig ist es, ob das Zweitveröffentlichungsrecht mehrfach ausgeübt werden darf oder sich mit der ersten Zweitverwertung erschöpft. (vgl. FN 411)

(8) Autoren, die das Zweitverwertungsrecht wahrnehmen, dürfen den Nutzern der Zweitpublikation keine Nutzungsrechte übertragen. Die so öffentlich zugänglich gemachten Werke können daher von den Autoren nicht unter eine freie Lizenz wie CC BY NC ( $\mathrm{NC}=$ nicht-kommerziell) gestellt werden.

(9) Das Recht kann von den Autorinnen und Autoren selbst wahrgenommen werden. Sie können aber auch eine Einrichtung, z. B. eine Bibliothek beauftragen, das Manuskript zum freien Abruf online zu stellen. ${ }^{396}$ Das Recht kann eine Bibliothek nicht von sich aus wahrnehmen.

(10) Das neue Recht gilt nicht rückwirkend, gilt also nur für Werke, die ab dem 1.1.2014 erschienen sind. Für diese allerdings auch, wenn vor diesem Termin über einen Publikationsvertrag exklusive Rechte an den Verlag abgetreten waren.

(11) Das Recht ist gegeben und anwendbar, ohne dass Einsicht in einen Vertrag oder sonstige Vereinbarungen genommen werden müsste.

(12) Das Recht kann erst nach einer einjährigen Sperr-/Embargofrist (die Zeit nach der Erstveröffentlichung) wahrgenommen werden.

(13) Das Recht gilt ausschließlich für das in § 19a geregelte Recht der öffentlichen Zugänglichmachung, also nicht für andere Verwertungsrechte, wie z. B. Verbreiten. Eine gedruckte Zweitveröffentlichung sieht die Regelung nicht vor.

(14) Bei einer Zweitverwertung muss auf die Quelle der Erstveröffentlichung referenziert werden.

(15) Die Zweitverwertung ist nur in der ,akzeptierten Autor-/Manuskriptversion“ erlaubt. Auf das Verlagslayout und das Verlagslogo muss verzichtet werden. Der Text sollte ansonsten jedoch inhaltsgleich mit der publizierten Verlagsversion sein.

(16) Das Zweitverwertungsrecht kann in keiner Weise abbedungen werden.

395 Im RefE zum Zweitveröffentlichungsrecht war das noch weiter gefasst: „im Rahmen einer mindestens zur Hälfte mit öffentlichen Mitteln finanzierten Lehr- und Forschungstätigkeit entstanden“https://bit.ly/2CHn4gx.

396 Vgl. die von der Europäischen EDV-Akademie des Rechts (EEAR) entwickelte Mustervereinbarung zur Einräumung eines einfachen Nutzungsrechts zum Zweck einer Zweitverwertung nach $\S 39,4$ in einem Repositorium - nachgewiesen unter: https://bit.ly/1BMOH3a. 
(17) Das Zweitverwertungsrecht gilt nicht für Werke, die in freier oder unternehmensbezogener wissenschaftlicher Tätigkeit entstanden sind, also außerhalb von öffentlich finanzierten Einrichtungen;

(18) Vermutlich gilt das Zweitverwertungsrecht auch nicht für Wissenschaftler, die in Forschungseinrichtungen des Bundes oder der Länder arbeiten - sie können schon nicht für die Erstveröffentlichung selbstständig verfügen.

(19) Nach dem Wortlaut des Gesetzes gilt das Zweitverwertungsrecht für Werke, die „im Rahmen einer mindestens zur Hälfte mit öffentlichen Mitteln geförderten Forschungstätigkeit entstanden" sind.

Entsprechend der Begründung der Bundesregierung sollte dieses Recht entweder nur für wissenschaftliche Artikel von Autorinnen und Autoren an Hochschulen gelten, deren Arbeiten mindestens zur Hälfte mit öffentlicher Projektförderung (z. B. DFG-, BMBF-, EU-Projekte, staatlich finanzierte Stipendien) finanziert wurden oder für wissenschaftliche Artikel, die von Mitarbeitern öffentlich finanzierter, außeruniversitären Forschungseinrichtungen (z. B. WGL, HGF, MPG) erstellt wurden. Das Zweitverwertungsrecht soll also nicht für Werke gelten, die aus der grundfinanzierten Forschung entstanden sind - erst recht nicht für Werke, die außerhalb von öffentlichen Einrichtungen entstanden sind (ausführlich in Abschnitt 11.3.4). Die obige lange Liste macht deutlich, dass zwar mit §38 Abs. 4 einige Fortschritte gegenüber §38, Abs. 1-3 erzielt wurden, dass aber das Recht stark eingeschränkt wurde. Trotzdem wurde von Seiten der Verlagswelt im Vorfeld Intensiv, mit fast schon dystopischen Argumenten dagegen opponiert. ${ }^{397}$

\subsubsection{Kritik an den Regelungen von $§ 38$ Abs. 4}

Die in der obigen Liste angeführten Einschränkungen stellen die Nützlichkeit der neuen Regelungen in Frage. Der Zeitraum (das Embargo), in dem nach der Regelung von $\S 38$ Abs. 4 das Verwertungsrecht zusätzlich an den Autor zurückfällt

397 Der Börsenverein des Deutschen Buchhandels „,ieht hier eine unzulässige Beschneidung der Verlagsrechte“. In seiner Stellungnahme hieß es: „Die Schaffung eines solchen Rechts würde dazu führen, dass in Deutschland anstelle nachhaltiger Strukturen für Open Access-Publikationen, die der Wissenschaft dienen, eine kostenträchtige und ineffiziente Repositorienlandschaft entstünde; würde deutsche Verlage im Wettbewerb mit ausländischen Verlagshäusern benachteiligen; bringt die Gefahr mit sich, dass geistes- und sozialwissenschaftliche Datenbanken und Zeitschriften in deutscher Sprache allmählich verschwinden; liefe auf eine entschädigungslose Enteignung der Leistungen deutscher Verlage hinaus und begegnet auch darüber hinaus europaund verfassungsrechtlichen Bedenken.“ Entsprechend die Forderung des Börsenvereins: „Auf die vorgeschlagene Regelung sollte deshalb ersatzlos verzichtet werden“ - Quelle buchreport 6. März 2013 https://bit.ly/2k8pMGz. 
(12 Monate), ist für Bildung und Wissenschaft nicht praktikabel, vor allem nicht in Fächern mit einem sehr raschen Erkenntnisumsatz. Die ersten Monate nach einer Publikation sind entscheidend für Zitierungswahrscheinlichkeit. Eine Flexibilisierung der Embargofrist wäre wegen der sehr unterschiedlichen Nutzungsformen in den verschiedenen Disziplinen die bessere Lösung gewesen. Auch der Bundesrat bedauerte in seiner Stellungnahme vom 20.9.2013 (also nach der Verabschiedung im Bundestag), ${ }^{398}$ dass sowohl die lange Embargofrist als auch die Beschränkung auf Zeitschriften hinter den Stand der Diskussion in der EU zurückgefallen sind. Überhaupt nicht zeitgemäß ist die Ausklammerung von Artikeln, die in nicht periodisch erscheinenden Sammlungen wie Konferenz-Proceedings, Festschriften, Sammelbänden etc. erschienen sind. In vielen MINT/STM-Fächern, vor allem in informatik-nahen Fächern, spielen Artikel in Konferenz-Proceedings eine größere Rolle als die in Zeitschriften.

Ebenso bleibt das in $\S 38$ Abs. 3 angesprochene Zweitverwertungsrecht für Zeitungsartikel wegen des „wenn nicht... “ für Journalisten faktisch versagt, auch für Journalisten im Wissenschaftsbereich. Hier wird es erst recht die Regel sein, dass Verlage in ihren Verträgen mit den Autoren (Journalisten) das exklusive Nutzungsrecht für sich reklamieren. $§ 38$ Abs. 4 hat das nicht korrigiert. Wenig zukunftsweisend ist in $\S 38$ Abs. 4 auch, dass Monographien von dem Zweitverwertungsrecht ausgeschlossen sind. In der „Begründung“ wird ausdrücklich darauf verwiesen, dass das Zweitverwertungsrecht den Autoren die Rechtssicherheit geben wird, „,ihre Publikationen im Wege des Open Access (zweit)zu veröffentlichen“. Schon 2013 war absehbar, dass Open Access auch auf größere Texte wie Monographien abzielt. Eine vorsichtige Übergangslösung oder Öffnungsklausel für neue Werktypen wäre sinnvoll gewesen.

Dass die Zweitverwertung auf die Manuskriptversion des Autors beschränkt wird, hat vor allem in den MINT-Fächern keinen gravierenden Nachteil, da wortwörtliche Zitate dort kaum gebräuchlich sind. Das ist aber vor allem in den Geisteswissenschaften anders, ${ }^{399}$ bei denen korrektes Zitieren (also nicht bloß Referenzieren) ein zentrales Erfordernis ist. Dieses Zugeständnis gegenüber den Verlagen war zudem unnötig. Unnötig, da das Zeitschriftengeschäft auch bei einer Zweitverwertung über die publizierte Verlagsversion nicht beeinträchtigt würde, da dieses ohnehin so gut wie vollständig über die Bibliotheken läuft, die im ersten Jahr der Embargofrist ihre Einkäufe bzw. Lizenzverträge schon abgeschlossen haben. Das vom Gesetzgeber angestrebte Ziel, Open Access über die Zweitverwertung durch

398 Bundesrat Drucksache 643/13 vom 20.9.2013 - https://bit.ly/2kA22Lv.

399 Das gilt sicherlich auch für die Rechtswissenschaft; vgl. (Wildgans 2019) Zuckerbrot oder Peitsche? 
die Autoren zu befördern, wird auch dadurch verfehlt, ${ }^{400}$ dass das zweitveröffentlichte Werk nicht über eine freie Lizenz freigestellt werden kann - über CC BY sowieso nicht, da eine gewerbliche Nutzung in $\S 38$ Abs. 4 untersagt ist; aber auch jede andere freie Lizenz, die mit den Zielen der Berliner Open-Access-Erklärung verträglich ist, wird durch $\S 38$ Abs. 4 behindert bzw. unmöglich gemacht. Im Zusammenhang der makroökonomischen Sicht auf das Zweitverwertungsrecht wird im folgenden Unterabschnitt auf das in der Liste oben unter (18) angesprochene Problem eingegangen, ob die grundfinanzierte Hochschulforschung durch $\S 38$ Abs. 4 ausgeschlossen ist oder, über eine kreative Interpretation, es ihr doch zugestanden wird.

\subsubsection{Ausklammerung der grundfinanzierten Hochschulforschung vom Zweitverwertungsrecht?}

Im Gesetz steht, dass das Zweitverwertungsrecht sich auf wissenschaftliche Beiträge in Periodika bezieht, „die überwiegend mit öffentlichen Mitteln finanziert wurden“. Das scheint eindeutig zu sein, sofern „überwiegend“ als mit „mehr als $50 \%$ “ verstanden wird. Eine institutionelle Ausdifferenzierung ist aus dem Text des Gesetzes nicht erkennbar. Auch im RefE des BMJV war keine solche Differenzierung zu erkennen. ${ }^{401}$ Zwischen Hochschulforschung und außeruniversitärer öffentlich finanzierte Forschung wurde nicht unterschieden. Umso mehr war die Fachöffentlichkeit, aber auch die politischen Institutionen außerhalb der Bundesregierung irritiert, dass diese Einheitlichkeit in der Begründung der Bundesregierung aufgekündigt wurde - und zwar mit einem makroökonomischen Argument. Die entsprechende Passage in der Begründung soll hier in voller Länge zitiert werden. Sie ist sozusagen ein zeitgeschichtliches und in Teilen bedrohliches Dokument. Das Zweitverwertungsrecht bezieht sich auf:

Forschungstätigkeiten, die im Rahmen der öffentlichen Projektförderung oder an einer institutionell geförderten außeruniversitären Forschungseinrichtung durchgeführt werden. Der Anwendungsbereich des Zweitveröffentlichungsrechts ist auf diese Bereiche beschränkt,

400 Kritik am Zweitverwertungsrecht wird wegen fehlende Orientierung an Open Access auch von (Wildgans 2019) geübt. Wildgans plädiert insgesamt für eine Öffnung von Open Access im juristischen Publikationsgeschehen und erwägt durchaus eine Mandatierung des Zweitverwertungsrechts auch im Sinne des (im Verfahren noch schwebenden) Konstanzer Vorstoßes (vgl. 11.3.6) zugunsten einer Open-Access-Mandatierung.

401 „Damit kommt der Regelungsvorschlag den nicht gewerblich handelnden Wissenschaftsinstitutionen (Universitäten, Forschungseinrichtungen) bzw. deren Repositorien zugute“ (so in der Begründung zum RefE - Beleg FN 395). 
da hier das staatliche Interesse an einer Verbreitung der Forschungsergebnisse besonders hoch ist. Anders als beispielsweise bei der rein universitären Forschung ist es üblich, dass der Staat bei der staatlichen Förderung Vorgaben hinsichtlich der Ziele und der Verwertung der Forschung macht. Diese Differenzierung lässt sich mit dem unterschiedlichen Gewicht des staatlichen Interesses an der Verbreitung und Verwertung der Forschungsergebnisse begründen. Die Projektförderung als auch die Tätigkeit an außeruniversitären Forschungseinrichtungen beruht auf programmatischen Vorgaben und Förderrichtlinien der Zuwendungsgeber, die damit den Erkenntnisgewinn in zuvor festgelegten Themenbereichen fördern wollen. Zu den Rahmenbedingungen dieser Förderbereiche gehören seit jeher Förderbestimmungen, die z. B. auch die Verwertung und Verbreitung der Ergebnisse regeln. Die erweiterten urheberrechtlichen Verwertungsmöglichkeiten sollen daher diesen Forschungsbereichen ermöglicht werden.

Zunächst einmal wird dadurch deutlich, dass das Zweitverwertungsrecht nur für Werke gilt, die über öffentliche Finanzierung entstanden sind. Im Vorfeld der Diskussion um das Zweitverwertungsrecht wurde diese sich abzeichnenden Beschränkung auf öffentlich finanzierte Wissenschaft kritisiert, z. B. in der Stellungnahme von GRUR zu §38 Abs. 4:

ob diese Privilegierung auf den Kreis der öffentlich finanzierten Urheber beschränkt bleiben kann oder ob der Gleichbehandlungsgrundsatz zu einer Erweiterung führen muss. Urheber, die eine geringere öffentliche Förderung oder gar keine Förderung erhalten haben, sondern die Aufwendungen für ihre Veröffentlichung überwiegend oder ganz selbst getragen haben, verdienen den Schutz eines Zweitveröffentlichungsrechts mindestens so sehr wie die Urheber, deren Rechtsstellung durch das vorgeschlagene Zweitveröffentlichungsrecht verbessert werden soll. Die Beschränkung des Entwurfs auf Veröffentlichungen aus einer überwiegend öffentlich finanzierten Lehr- und Forschungstätigkeit sollte deswegen gestrichen werden.

Diese von GRUR vorgeschlagene Ausweitung auf Forschung allgemein ist in der Tat sinnvoll, da es das primäre Ziel dieser Gesetzgebung gewesen war, das Interesse des individuellen Wissenschaftlers (auch außerhalb der öffentlichen Finanzierung) an möglichst breiter Sichtbarkeit zu befördern. ${ }^{402}$ Die oben zitierte Begründung der Bundesregierung ist aber noch in anderer Hinsicht bedenklich diskriminierend: Für die normale Hochschulforschung bestand für die damalige Regierung (Koalition CDU/CSU und FDP) offenbar kein besonderes Interesse an einer Verbreitung und Verwertung der dort erzielten Forschungsergebnisse. Die Politik geht weiter davon aus, dass der Staat für öffentliche Drittmittelprojekte, finanziert z. B. über die DFG, und für die Forschung in Einrichtungen wie z. B. Max-Planck, Helmholtz oder

402 Entsprechend wurde auch in der Enquete-Kommission „Internet und digitale Gesellschaft“ die Frage stellt, „ob nicht ein generelles Zweitverwertungsrecht für Urheber von wissenschaftlichen Beiträgen [also unabhängig von der öffentlichen Finanzierung - RK] unabhängig eingeführt werden sollte.“ Enquete Kommission 2011, S. 21. 
Leibniz, aber auch forschungsaktive Bundesanstalten für Ziele und Verwertung Vorgaben macht. Diesen geförderten Forschungsvorgaben liegen programmatische Vorgaben und Förderrichtlinien der Zuwendungsgeber zugrunde.

Im Bundestag wurde die oben ausführlich zitierte Begründung der Bundesregierung als verbindlich für das Zweitverwertungsrecht angesehen. Die Oppositionsparteien kritisierten allerdings die Privilegierung bestimmter Wissenschaftskreise zunächst schon bei der ersten Lesung ${ }^{403}$ und dann bei der Endabstimmung erneut scharf. ${ }^{404}$

Ein solches - exklusives - Zweitverwertungsrecht für bestimmte, von der Bundesregierung als besonders relevant erachtete Forschungskreise ist nicht nur verfassungsrechtlich bedenklich, sondern setzt zudem die Forschungsleistung von Wissenschaftlerinnen und Wissenschaftlern an deutschen Hochschulen herab. (René Röspel, SPD)

Das Zweitverwertungsrecht soll sich auf alle wissenschaftlichen Publikationen erstrecken, die überwiegend aus öffentlichen Mitteln finanziert worden sind. Zweitens. Eine Zweitveröffentlichung wird für alle Publikationsformen ermöglicht. (Petra Sitte, Die LINKE)

Auch der Bundesrat sprach bei seiner ersten Beratung des Gesetzentwurfs im Mai 2013 nicht nur von einer Zweiklassengesellschaft, sondern sogar von einer „Dreiklassengesellschaft“, die durch $\S 38$ Abs. 4 errichtet würde. Streng genommen ist es sogar eine Vierklassengesellschaft: außeruniversitäre Forschungseinrichtungen, drittmittelfinanzierte Forschung an Hochschulen, grundfinanzierte Forschung an Hochschulen und speziell Geistes- und Sozialwissenschaften an Hochschulen. Letztere haben traditionell niedrige Drittmittelquoten und würden damit von dem Zweitverwertungsrecht weitgehend ausgeschlossen. ${ }^{405}$ Entsprechend hatte der Bundesrat gefordert, dass das Zweitverwertungsrecht zumindest für alle öffentlich finanzierten Wissenschaftler gelten sollte. Er ist damit einer Empfehlung des Landes Baden-Württemberg gefolgt: „Für die Herausnahme des gesamten an Hochschulen beschäftigten wissenschaftlichen Personals gibt es keinen sachlichen Grund.“406 Auch in dieser Begründung steht das öffentliche Interesse mit der Begründung der öffentlichen Finanzierung im Vordergrund. Speziell den Geistesund Sozialwissenschaften damit gleichsam zu bescheinigen, dass sie politisch und ökonomisch irrelevant seien, war vermutlich auch durch die Bundesregierung nicht

403 Opposition im Bundestag. Protokoll 17/244, 7.6.2013 - https://bit.ly/2lD1WTE.

404 Auch bei der abschließenden 2. und 3. Lesung im Bundestag wurde dieser Ausschluss von den Vertretern der Oppositionsparteien (Bündnis90/DIE GRÜNEN, Linke, SPD) kritisiert. Sie gingen also weiter davon aus, dass die Begründung der Bundesregierung die verbindliche Auslegung von $\S 38$ Abs. 4 sei.

405 Vgl. (Kuhlen 2013b) Manifestierung der Dreiklassengesellschaft.

406 Bundesratsbeschluss 3.5.2013. Quelle: bildungsklick - https://bit.ly/30lfeUc. 
beabsichtigt. Warum ist die Ausklammerung der Geistes- und Sozialwissenschaften vom Zweitverwertungsrecht ohne Not überhaupt ins Spiel gebracht worden? Wenn schon kein ökonomischer Vorteil von der Politik davon erwartet wurde welcher Nachteil wäre aber bei einem Zweitverwertungsrecht für alle in Bildung und Wissenschaft Tätigen entstanden? Tatsächlich geht man wohl nicht fehl in der Annahme, dass die Ausklammerung der Geistes- und Sozialwissenschaften dem Lobbying deutscher Verlage geschuldet ist, die auf diesem Gebiet Zeitschriften betreiben.

Die kritische Einschätzung des Bundesrats zu diesen Gesetzesvorgaben des Bundes hat er in seinem Beschluss vom 20.9.2013 (Drucksache 643/13), also nach den abschließenden Lesungen im Bundestag, noch einmal bekräftigt. ${ }^{407}$ Seine Entscheidung, für das Zweitverwertungsgesetz den Vermittlungsausschuss dennoch nicht anzurufen, war auf einiges Unverständnis gestoßen. Eine Ablehnung bzw. Anrufung des Vermittlungsausschusses war wegen des Ausschlusses der grundfinanzierten Hochschulforschung von dem neuen Recht erwartet worden. ${ }^{408}$ Der Bundesrat löste dieses Problem dann auf eine auf den ersten Blick sibyllinisch anmutende Weise, indem er feststellte,

dass §38 Absatz 4 Satz 1 UrhG-neu, dessen Anwendungsbereich sich zumindest im Wege einer verfassungskonformen Auslegung auch auf das gesamte, an den Hochschulen beschäftigte wissenschaftliche Personal erstrecken muss, dem begünstigten Personenkreis ein vertraglich nicht abdingbares Recht auf Zweitveröffentlichung eröffnet. ${ }^{409}$

Der Bundesrat ignorierte also die oben zitierte Einschätzung der Bundesregierung und interpretierte das Gesetz wortwörtlich (,im Rahmen einer mindestens zur Hälfte mit öffentlichen Mitteln geförderten Forschungstätigkeit”) bzw. leitete er daraus die telelogische Interpretation ab, dass damit auch das gesamte wissenschaftliche Personal an Hochschulen das Zweitverwertungsrecht in Anspruch annehmen

407 Bundesrat Drucksache 643/13 vom 20.9.2013 - https://bit.ly/30qdQzC.

408 Auch das Aktionsbündnis Urheberrecht für Bildung und Wissenschaft war in einem weit verbreiteten und genutzten Flyer davon ausgegangen, dass Hochschullehrer nur dann vom Recht begünstigt seien, wenn ihre Werke im Zusammenhang von Drittmittelforschung entstanden waren: „Wissenschaftliche Artikel, die mit Grundmitteln einer Hochschule finanziert wurden, hat der Gesetzgeber von der Nutzung des Zweitveröffentlichungsrechts ausgeschlossen.“ Flyer zum Zweitveröffentlichungsrecht - https://bit.ly/2KOh9LS.

409 Es ist auch davon auszugehen, dass der Bundesrat es nicht darauf ankommen lassen wollte, das gesamte Paket der Reform scheitern zu lassen. Es war nicht vorgesehen, über die einzelnen Regelungen getrennt abzustimmen, also z. B. die Regelung für verwaiste Werke zu billigen, aber die für das Zweitverwertungsrecht abzulehnen bzw. dafür den Vermittlungsausschuss anzurufen. Kurz vor Ende der Legislaturperiode wäre es bei Anrufung des Vermittlungsausschusses aus zeitlichen Gründen nicht mehr möglich gewesen, das Gesetz gültig werden zu lassen. 
könne. Inzwischen haben sich auch die meisten Urheberrechtskommentare dieser Auffassung tatsächlich angeschlossen. In den Kommentaren zu §38 Abs. 4 wurde deutlich, dass für die telelogische Auslegung eines Gesetzes der Wille des Gesetzgebers, also in diesem Fall des Bundestags, entscheidend sei und nicht die zwar eindeutig formulierte Intention der das Gesetz zur Entscheidung vorlegenden Bundesregierung. ${ }^{410}$ Allerdings handelt es sich bei dieser Einschätzung bislang nur um eine Auslegung aus der Rechtswissenschaft. Bislang hat es keine Klage gegen das Gesetz gegeben. Entsprechend haben die Gerichte auch keine Gelegenheit gehabt, den Widerspruch zwischen Intention der Bundesregierung und Wortlaut des Gesetzes aufzulösen. Die Protokolle der ersten und dann der zweiten und dritten Lesung sind vermutlich nicht eindeutig so $\mathrm{zu}$ lesen, dass sich der Bundestag bei seinen Beratungen der Interpretation des Bundesrats angeschlossen habe. Alle Oppositionsparteien haben, wie oben zitiert, in ihren hinterlegten Stellungnahmen die Ausklammerung der grundfinanzierten Hochschulforschung stark kritisiert. Die entsprechenden eingebrachten drei Anträge der SPD, Bündnis90/Die GRÜNEN und DIE LINKE wurden von der Mehrheit der Regierungskoalition abgelehnt. Angesichts der großen Auslegungsspielräume der Gerichte kann es nicht ausgeschlossen werden, dass vor Gericht die Ablehnung der Oppositionsanträge doch als „Unterstützung“ des Vorschlags bzw. hier der Begründung der Bundesregierung durch die Mehrheit des Bundestags gewertet wird. Am Ende von 11.3.6 wird auf das Problem der Extension des Zweitverwertungsrechts am Beispiel der Klage gegen die Universität Konstanz, eine Zweitverwertungsverpflichtung in der Satzung vorzusehen, eingegangen.

\subsubsection{Ein einfaches Nutzungsrecht}

Der Gesetzgeber hätte sich die gesamte Debatte um das Zweitveröffentlichungsrecht sparen können, wenn er eine urhebervertragsrechtliche Lösung gewählt hätte, dass in Verträgen zwischen Autoren und Urhebern grundsätzlich nur einfache Nutzungsrechte vereinbart werden dürfen - vielleicht mit der Einschränkung, dass dem Verlag dafür exklusiv für eine festzulegende Zeit die kommerzielle einfache Verwertung zugestanden wird. Dies könnte dazu beitragen, eine Symmetrie in der Rechten zwischen Urheber und Verwerter herzustellen. Bislang sehen die

410 So (Peukert 2017) Kommentar zu § 38 Abs. 4, RN 45-47: „Der Entstehungsgeschichte des Gesetzes [könne] nicht entnommen werden, dass der Bundestag bei der Verabschiedung des Gesetzes den gesamten Bereich der grundständigen Hochschulforschung, die einen sehr erheblichen Teil, wenn nicht die Mehrzahl der öffentlich geförderten wissenschaftlichen Beiträge in der Bundesrepublik hervorbringt, ausgrenzen wollte.“ 
Verträge für die Nutzungsrechte der Verleger vor, dass die Nutzung auf mehrfache Weise geschehen kann. Der Verlag besitzt also dadurch auch ein Zweit- bzw. Vielfachverwertungsrecht. Dieses mehrfache Recht wird den Urhebern durch $\S 38$ Abs. 4 nicht zugebilligt. Das Zweitverwertungsrecht darf vom Urheber, anders als vom Verleger, nur „einmalig“ genutzt werden. ${ }^{411}$ Wenn Urheber ein nur einmal in Anspruch zu nehmendes Zweitverwertungsrecht haben, ist es dann gerechtfertigt, dass Verlage das Vielfachverwertungsrecht haben? Das ist schwer einzusehen Verlage sollten immer nur ein einfaches Nutzungsrecht erwerben können und dies auch nur für den Zweck der ersten Verwertung. Sind weitere Verwertungen vorgesehen, so müssten neue Vereinbarungen mit dem Urheber getroffen werden. Eine Beschränkung auf ein einfaches kommerzielles Nutzungsrecht wäre eine politische Entscheidung gewesen, die aber in keiner Weise gegen geltendes Recht oder gar gegen Grundrechte verstoßen würde. Dabei ist der Vorschlag, die Nutzungsrechte der Verwerter auf einfache Nutzung zu beschränken, keineswegs eine revolutionäre Forderung. Schon im Juli 2001 hatte der Wissenschaftsrat in seinen Empfehlungen zur digitalen Informationsversorgung durch Hochschulbibliotheken ${ }^{412}$ einen konkreten Vorschlag für eine Zweitverwertung gemacht, der in der Konsequenz auch auf ein nur einfaches Verwertungsrecht für Verlage hinausläuft:

\footnotetext{
Digitale wissenschaftliche Publikationen sollten durch eine hohe Verfügbarkeit und Zugänglichkeit gekennzeichnet sein. Deshalb ist der Wissenschaftsrat der Auffassung, daß Autoren ihre Verwertungsrechte mit der Freigabe zur wirtschaftlichen Verwertung nicht pauschal an Verlage abtreten und diesen ein exklusives Recht für sämtliche Arten der Verwertung einräumen sollten. [fett durch RK] Von den Wissenschaftlern in ihrer doppelten Funktion als Autoren und Nutzer erwartet der Wissenschaftsrat ein differenziertes Umgehen mit dem Urheberrecht. Im Wissenschaftssystem sollte Einvernehmen bestehen, daß dem Autor das Recht zur Zweitverwertung erhalten bleiben sollte, um eine elektronische Neuauflage (Re-Print) für die Möglichkeit einer unabhängigen Online-Veröffentlichung beispielsweise über den Server einer Hochschule oder über Fachportale anzubieten. Vom Autor ist dabei ein entsprechender Hinweis vorzusehen, an welchem Ort und in welcher Form das Dokument erstmals bei einem Verlag veröffentlicht worden ist.
}

Gegen die Begrenzung auf ein einfaches kommerzielles Nutzungsrecht läuft allerdings die Mehrheit der Juristen als Einschränkung von Vertragsfreiheit Sturm. Diese juristische Weigerung, die sich der Gesetzgeber zu eigen gemacht hat, ist dem fehlenden Mut zuzurechnen, den gegebenen großen Gestaltungsspielraum zu nutzen, um neue Wege im Urheberrecht begehbar zu machen.

411 Vgl. (Bruch/Pflüger 2014) Das Zweitveröffentlichungsrecht, RN393.

412 Wissenschaftsrat. Drs. 4935/01 Greifswald, 13. Juli 2001 -https://bit.ly/2Zb3Spi, Zitat S. 26. 


\subsubsection{Mandatierung - besser ein zusätzliches institutionelles Zweitverwertungsrecht?}

Die Diskussion um die Regelung für das Zweitverwertungsrecht hat deutlich gemacht, dass das öffentliche Interesse an einer umfassenden Nutzung des mit öffentlichen Mitteln produzierten Wissens nicht so befördert wird, wie es nötig und möglich ist. Der Schwerpunkt lag für den Gesetzgeber darin, die Interessen des Urhebers zu stärken. Beide Interessen sollten aber auch in der gesetzlichen Regelung zusammen gesehen werden. Ein Zweitverwertungsrecht, das nur abstrakt die Autonomie der Urheber stärkt und das, so wie es konzipiert ist, nicht von den Urhebern angenommen wird, geht doppelt fehl. Es verfehlt das individuelle Autonomieziel und verfehlt das anvisierte Ziel, der Öffentlichkeit (darunter auch allen Wissenschaftlern) einen freieren Zugang zum publizierten Wissen zu verschaffen - freier gegenüber der Nutzung der Erstveröffentlichung in kommerziellen kostenpflichtigen Zeitschriften.

In diesem Abschnitt wird vorgeschlagen, die Orientierung an dem individuellen Interesse durch ein institutionelles Zweitverwertungsrecht zu ergänzen. Tatsächlich wird das Gesetz ausdrücklich nicht nur durch das individuelle Interesse, sondern auch durch das öffentliche Interesse und auch das der Wirtschaft an der freien Verfügung publizierter Werke begründet: „Nur wenn Forschungsergebnisse frei verfügbar sind, können sie Grundlage weiterer Forschungsaktivitäten sein und die damit verbundenen positiven gesamtwirtschaftlichen Effekte auslösen. Forschung ist somit kein Selbstzweck. ... [durch das Gesetz werde der] freie Zugang zu wissenschaftlichen Informationen gefördert“ (aus der Begründung des RegE). „Wenn das Ziel darin liegt, die öffentlich finanzierte Forschung der steuerzahlenden Öffentlichkeit leicht zugänglich zu machen“, so Steinhauer in seinem Beitrag in Legal Tribune Online, ${ }^{413}$ „warum wurde die Zweitverwertung dann nicht verpflichtend vorgeschrieben?“ Dies aber haben Bundesregierung und dann die Mehrheit im Bundestag abgelehnt. In der Begründung für $\S 38$ Abs. 4 wird unmissverständlich festgehalten, dass das Zweitverwertungsrecht nur ein Recht sei und keine Zweitverwertungsverpflichtung. Ebenso wird in der Begründung der Bundesregierung deutlich, dass das Zweitverwertungsrecht nur als ein individuelles Autorenrecht anzusehen sein. Dieses Recht werde durch „die grundrechtlich geschützte Position der Wissenschaftler aus Artikel 5 Absatz 3 und Artikel 14 Absatz 1 des Grundgesetzes auf urhebervertragsrechtlicher Ebene“ begründet. Wissenschaftsfreiheit würde, so die Bundesregierung, durch $\S 38$ Abs. 4 sogar bekräftigt.

413 (Steinhauer 2013) Mehr Open Access oder bloßer Placebo?; vgl. dazu (Kuhlen 2010d): Kommentar zu Steinhauer: Das Recht auf Sichtbarkeit - https://bit.ly/2MvsO4h - ausführlich: Ein Recht auf Sichtbarkeit - aber nicht auch ein Recht auf Sichtbarwerden? - https://bit.ly/2VKJ3hL. 
Der deutsche Gesetzgeber hat sich allerdings in der Begründung zu §38 Abs. 4 mit Blick auf Mandatierung etwas „,vorgewagt“, nämlich dass dem „staatlichen Interesse an der Verbreitung und Verwertung der Forschungsergebnisse“ über die „Förderrichtlinien der Zuwendungsgeber“ (z. B. der DFG) entsprochen werden könnte. Es solle den öffentlichen Förderinstitutionen wie die DFG überlassen bleiben, über eine Verpflichtung zur Wahrnehmung des neuen Rechts durch die Urheber bzw. über ein institutionelles Recht zu entscheiden. „Zu den Rahmenbedingungen dieser Förderbereiche gehören seit jeher Förderbestimmungen, die z. B. auch die Verwertung und Verbreitung der Ergebnisse regeln.“414 Das ist auch seit einigen Jahren der Trend in der internationalen Förderpolitik, erkennbar über verschiedene Erklärungen aus der EU bis hin zu Horizon 2020. Alle Mitgliedsstaaten werden ermutigt, alle öffentlich finanzierten Forschungsergebnisse in den öffentlichen Raum zu stellen. ${ }^{415}$

Zur Wissenschaftsfreiheit eines jeden Wissenschaftlers gehört es, darüber $z u$ entscheiden, ob, wann und wie er sein Werk veröffentlichen will. Durch das jetzige Zweitverwertungsrecht über $\S 38$ und insbesondere über $\S 38$ Abs. 4 ist dieses Recht als Erstveröffentlichungsrecht überhaupt nicht betroffen. ${ }^{416}$ Kein Wissenschaftler soll zu einer Entscheidung über das ob, wann und wie zu veröffentlichen gezwungen werden. Es wird daher auch hier nicht für zielführend gehalten, Wissenschaftler dazu zu verpflichten, sie zu zwingen, dieses Zweitveröffentlichungsrecht nun auch tatsächlich wahrzunehmen. Das heißt aber nun keineswegs, dass die Zweitveröffentlichung gänzlich ins Belieben des Urhebers gestellt werden sollte. Mandatierung als Zwang ist kein Mittel zur Steuerung wissenschaftlichen Handelns. Rechtsetzung und Rechtsprechung in Deutschland,

414 Vgl. (Kretschmer 2010) Wo geforscht wird, fallen Früchte.

415 European Research Council: Scientific publication: Policy on Open Access. https://bit.ly/ 2jYLJCm; EU-Commison 2012: "encouraged all EU Member States to put public-funded research results in the public sphere in order to make science better and strengthen their knowledge-based economy"; die entsprechende Empfehlung der EU Commission 2012 - https://bit.ly/1eCRmRw.

416 Daher geht die immer wieder geäußerte Kritik am Zweitverwertungsrecht ins Leere. Erwähnt sei nur der Präsident des DHV, Professor Dr. Bernhard Kempen, der 2010 anlässlich des 60. DHV-Tages in Hamburg kritisiert hatte, dass das von der „Allianz der deutschen Wissenschaftsorganisationen“ geforderte Zweitveröffentlichung Wissenschaftler verpflichte, „ihre Werke kostenlos zu veröffentlichen. [...] Damit würde sowohl das Urheberrecht als auch die Wissenschaftsfreiheit der betroffenen Wissenschaftler untergraben. [...] Ausschließlich sie [die Wissenschaftler] selbst müssten entscheiden, ob sie ihre Werke im Rahmen von Open Access-Publikationen kostenlos zur Verfügung stellten. [...] Für eine Verpflichtung zur Open Access-Publikation kann insbesondere nicht die aus öffentlichen Mitteln geförderte Vergütung bzw. Besoldung von Wissenschaftlerinnen und Wissenschaftlern als Begründung herhalten.“ (Bernhard Kempen: Wissenschaftler müssen allein entscheiden, ob, wann und wo publiziert wird. Pressemitteilung des DHV vom 23.03.10). 
aber auch Förderorganisationen wie die DFG, tun sich nach wie vor mit einer Mandatierung schwer, selbst nur für eine Zweitverwertung, erst recht für eine Erstveröffentlichung. ${ }^{417}$ Anders in vielen anderen Ländern wie z. B. in der Schweiz. Dort gilt, dass öffentlich finanzierte Werke (einschließlich Datensammlungen) „ein öffentliches Gut [sind] und daher in elektronischer Form zeitnah und kostenlos zugänglich sein und für die Wiederverwendung durch Dritte zur Verfügung stehen“ sollten. Für Publikationen, die aus den Schweizer Akademien entstanden sind, müssen alle „Publikationen sofort, kostenlos und frei zur Verfügung (Diamond Open Access)“ gestellt werden. ${ }^{418}$ Werke, deren Entstehung über den Schweizerischen Nationalfonds zur Förderung der wissenschaftlichen Forschung (SNF) finanziert wurden, müssen ebenfalls frei für die Öffentlichkeit zugänglich gemacht werden. ${ }^{419}$ Falls dies nicht über eine Open-Access-Erstpublikation erfolgt, müssen Zeitschriftenaufsätze über den grünen Weg (Zweitpublikation) spätestens nach 6 Monaten, Bücher nach 12 Monaten zugänglich gemacht werden. Bis Mitte 2019 waren allerdings nur $48 \%$ der so geförderten Werke verfügbar gemacht worden. Um $100 \%$ zu erreichen, geht der SNF dazu über, die von ihm geförderten Forscher mahnend direkt anzuschreiben.

Auch sonst kommt in der wissenschaftlichen Gemeinschaft Bewegung in der Frage der Mandatierung. Das “encouraged” bzw. „requested“ wird immer

417 Der deutsche Gesetzgeber hat sich allerdings in der Begründung zu §38 Abs. 4 mit Blick auf Mandatierung etwas „,vorgewagt“, nämlich dass dem „staatlichen Interesse an der Verbreitung und Verwertung der Forschungsergebnisse “über die „Förderrichtlinien der Zuwendungsgeber“ (z. B. der DFG) entsprochen werden könnte. Es solle den öffentlichen Förderinstitutionen wie der DFG überlassen bleiben, über eine Verpflichtung zur Wahrnehmung des neuen Rechts durch die Urheber bzw. über ein institutionelles Recht zu entscheiden. „Zu den Rahmenbedingungen dieser Förderbereiche gehören seit jeher Förderbestimmungen, die z. B. auch die Verwertung und Verbreitung der Ergebnisse regeln. " Das ist auch seit einigen Jahren der Trend in der internationalen Förderpolitik, nicht zuletzt über verschiedene Erklärungen aus der EU bis hin zu Horizon 2020 (European Research Council: Scientific publication: Policy on Open Access - https://bit.ly/2jYLJCm; (EU-Commison 2012): "encouraged all EU Member States to put public-funded research results in the public sphere in order to make science better and strengthen their knowledge-based economy"; die entsprechende Empfehlung der EU Commission 2012 - https://bit.ly/1eCRmRw.

418 Creative Commons stellt zur Realisierung von Diamond Open Access eine CC0-Lizenz bereit, die Autoren, die ihr Werk gänzlich in die Public domain stellen wollen, die Möglichkeit eröffnet, "to opt out of copyright and database protection, and the exclusive rights automatically granted to creators the 'no rights reserved' alternative to our licenses." creativecommons.org - https://bit.ly/2x9IalG. Dabei sind sich die für CC bzw. CCO zuständigen Personen vielleicht nicht bewusst (oder sie ignorieren es), dass manche Urheberrechtsgesetze, wie z. B. das in Deutschland, es gar nicht erlauben, Persönlichkeitsrechte aufzugeben.

419 Schweizerischer Nationalfonds: Auf dem Weg zu $100 \%$ : Steigerung bei Open-Access-Publikationen notwendig - https://bit.ly/31hxLAY. 
mehr durch ein "required" ersetzt. Durch (derzeit) 17 nationale und internationale Forschungsförderer und Forschungsorganisationen, unterstützt durch die EU-Kommission und den Europäischen Forschungsrat, wurde im Oktober 2018 die Initiative cOAlition mit Plan S gegründet. ${ }^{420}$ Plan S fordert, ${ }^{421}$ dass ab 2021 jede Forschung, die von „public or private grants provided by national, regional and international research councils and funding bodies" gefördert wird, in OpenAccess-Zeitschriften oder über Open-Access-Repositories publiziert bzw. sofort (“immediately") zugänglich gemacht wird. Dieses Ziel soll Anfang 2021 zumindest für Werke aus öffentlich finanzierter Forschung vollständig erreicht werden.

Allerdings hat offenbar ein großer Teil der Wissenschaftler, zumindest in Deutschland, ein Problem mit einer solchen Mandatierung. Vielleicht liegt es auch an der Begrifflichkeit. Wer will schon mandatiert werden? Zudem - wie sollte ein solcher Zwang, wie sollte eine Mandatierung von Wissenschaftlern zur Wahrnehmung des Zweitverwertungsrechts realisiert werden? Soll er ein Jahr nach der Erstveröffentlichung zu seiner Bibliothek gehen und ihr mitteilen, dass er jetzt der Bibliothek das Werk zur Zweitveröffentlichung über ihr Repository zur freien Nutzung für jedermann übergeben möchte? Das ist eine unrealistische Situation. Für Wissenschaftler ist der Veröffentlichungsprozess i.d. R. damit abgeschlossen, dass sein Werk, möglichst prominent platziert, publiziert und im Prinzip der Fachöffentlichkeit zugänglich gemacht ist. Weitere Veröffentlichungsinitiativen werden eher als lästig empfunden, die von einer weiterführenden Forschungsarbeit ablenken. Höchstens wird dem weiteren Interesse dadurch entsprochen, dass, wie oben erwähnt, die jeweilige Publikation auf der eigenen Website zumindest als Referenz angezeigt, aber auch immer mehr, und auch immer mehr toleriert von den Verlagen, in der Autorenvolltextversion zum Download bereitgestellt wird. Jedoch sollte die ergänzende Veröffentlichung nicht vom good will der kommerziellen Verwerter abhängig gemacht werden - erst recht nicht von Einschränkungen über Form und Zeitpunkt der Freigabe. Fraglich, ob durch Selbstpublikation auf der eigenen Website die nachhaltige Verfügung über publiziertes Wissen, also auch für nachfolgende Generationen, gewährleistet werden kann - auch kommerzielle Informationsdienstleistungen bleiben nicht unbedingt nachhaltig verfügbar. Ein anderer Weg hätte gefunden werden müssen. Leider hat die Bundesregierung offenbar, anders als bei vergriffenen Werken, nicht erwogen, den die Urheber tragenden Organisationen, in Ergänzung zu dem persönlichen Recht der Urheber,

420 cOAlition S - https://www.coalition-s.org/; vgl. Science Europe - https://bit.ly/2k6i7IG.

421 Plan S Principles, konkretisiert in 10 zentralen Zielen - https://bit.ly/2W7f9AM. Plan S, wie insgesamt die cOAlition, wird von Science Europe (https://www.scienceeurope.org/) koordiniert und wird von zahlreichen nationalen Förderorganisationen und anderen internationalen Einrichtungen unterstützt. 
ein institutionelles Recht zur Zweitverwertung zuzugestehen. Dafür sah sie offenbar keinen Gestaltungsspielraum im geltenden Urhebervertragsrecht.

Ein institutionelles Zweitverwertungsrecht ist das Recht (und die daraus folgende Pflicht) der die Autoren tragenden Institutionen, die Werke ihrer Forscher zweitzuveröffentlichen und damit für alle unter einer freien Lizenz zugänglich zu machen. Als Speicher- und Zugriffsmedium bieten sich die ohnehin in Bibliotheken so gut wie immer schon eingerichteten institutionellen Repositories an. Andere Repository-Formen wie fachspezifische oder nationale bzw. internationale könnten durch entsprechende Kooperationsformen zustande kommen. Ein institutionelles Zweitverwertungsrecht ist keine Einschränkung von Wissenschaftsfreiheit, weder in negativer noch in positiver Hinsicht. Das negative Publikationsrecht ist das Recht, nicht zum Publizieren/Veröffentlichen gezwungen zu werden; das positive Publikationsrecht ist das Recht zu entscheiden, ob, wann und wie ein Werk zu veröffentlichen. ${ }^{422}$ Diese Publikationsrechte bleiben mit Blick auf die Erstveröffentlichung uneingeschränkt gewahrt. Wie ist es nachzuvollziehen, dass das Urhebervertragsrecht den kommerziellen Verlegern eine Vielzahl von Verwertungsmöglichkeiten, über die primäre Publikation hinaus, zubilligt, aber Vermittlungsorganisationen wie Bibliotheken nicht das Recht gibt, die erstveröffentlichten Werke der in der entsprechenden Organisation arbeitenden Wissenschaftler zweitzuveröffentlichen?

Ob dazu der Weg der Beste ist, dem das Land Baden-Württemberg vorgesehen bzw. für den sich dann die Universität Konstanz entschieden hatte, ist in rechtlicher Hinsicht noch nicht abschließend geklärt. ${ }^{423}$ Das Land hatte versucht, die Wissenschaftler in den Hochschulen des Landes auf eine Wahrnehmung des Zweitverwertungsrechts zu verpflichten. Dazu wären dann die an den Hochschulen eingerichteten Repositories geeignet. Das Land verstand das nicht als Urheberrechtsregelung (Urheberrecht ist Bundesrecht), sondern als Änderung der hochschulrechtlichen Vorschriften, konkret über § 44 Abs. 6 LHG, als ,dienstrechtliche Umsetzung“ des Urheberrechtsgesetzes im Rahmen der Zuständigkeit des Landes Baden-Württemberg. ${ }^{424}$ Diese Vorgabe durch die Landesgesetzgebung

422 Allerdings gibt es Ausnahmen vom positiven Publikationsrecht: Wissenschaftler, die in der Wirtschaft arbeiten, genießen den Schutz der Wissenschaftsfreiheit. Dennoch kann ihnen das positive Publikationsrecht durch ihren Arbeitgeber verweigert werden. Das Gleiche gilt - so problematisch das zu sein scheint - auch für Wissenschaftler in Forschungsinstitutionen des Bundes und der Länder. Und Dissertationen müssen - so jedenfalls die Promotionsordnungen öffentlich zugänglich gemacht werden, damit der Doktorand seinen Titel tatsächlich beanspruchen kann.

423 Ausführlich dazu (Hartmann 2017) Zwang zum Open Access-Publizieren?

424 „Angehörige des wissenschaftlichen Personals der Hochschulen sind verpflichtet, sich das Recht auf nicht-kommerzielle Zweitveröffentlichung in der Regel binnen einer Frist von sechs 
wurde allerdings von vielen Seiten bei einer Anhörung im Landtag kritisiert, mit der Konsequenz, dass nun die Universitäten das selber durch Änderung ihrer Satzung regeln sollten. Konstanz war die erste Universität, die das tat. Dagegen klagten Professoren aus der Juristischen Fakultät. ${ }^{425}$ Darüber ist in der Sache bis heute nicht entschieden. Ob die Angelegenheit bis vor das BVerfG kommt, ist offen offen auch, wie sich das BVerfG im Falle der Klage gegen die Hochschulsatzung der Universität Konstanz entscheiden würde. Offen ist schließlich auch, ob das formale Argument des vorausgegangenen Münchener OLG - nämlich dass die Regelung in Konstanz einen unzulässigen Eingriff in die Urheberrechtskompetenz des Bundes darstellt - als entscheidend für die Ablehnung des gesamten quasi institutionellen Zweitverwertungsrechts herhalten muss.

Ausführlich setzen sich Höpfner/Amschewitz ${ }^{426}$ mit der Klage (genauer: ein Normenkontrollantrag) gegen die in die Satzung der Universität Konstanz vorge-

Monaten nach der Erstveröffentlichung vorzubehalten, wenn es sich um Publikationen von wissenschaftlichen Erkenntnissen in periodisch erscheinenden Sammlungen und Zeitschriften handelt, die im Rahmen der Dienstaufgaben gewonnen worden sind. Sie können durch Rechtsverordnung des Wissenschaftsministeriums dazu verpflichtet werden, die Zweitveröffentlichung in hochschuleigene Repositorien einzustellen; auf $\S 28$ Absatz 3 wird verwiesen.“ (Entwurf § 44 Abs. 6, LHG)

425 Der Text der Klageschrift ist bislang nicht öffentlich gemacht. Öffentlich ist allerdings eine Stellungnahme von Christoph Schönberger, C., öffentliches Recht an der Universität Konstanz, einer der Kläger, im Deutschlandfunk vom 25.1.2017 - https://bit.ly/2Z8vsTx. Diese Stellungnahme geht in Teilen an der Sache vorbei: „Wir entscheiden ja bis jetzt selbst, in welchen Publikationsformaten, in welchen Zeitschriften, wir unsere Ergebnisse publizieren. Und nach unserer Meinung gehört es zu unserer Wissenschaftsfreiheit, dass auch selbst entscheiden zu können.“ Diese Entscheidung ist weder durch $\S 38$ Abs. 3 noch durch die Konstanzer Satzung beeinträchtigt. Die Erstpublikation bleibt in der Gänze im Veröffentlichungsrecht des Urhebers. Auch die andere Aussage: „In dem Augenblick, in dem wir gezwungen werden, die Ergebnisse in irgendeiner Form auf einem Server bereit zu legen, geben wir diese Hoheit über diese Forschungsergebnisse, über die Art, wie wir sie präsentieren wollen, auf. Das möchten wir nicht“ kann kaum überzeugen. Schließlich wird die Arbeit auf dem Server in einer Form repräsentiert, die als Wissensobjekt vom Autor erstellt wurde. Anders als Schönberger unterstützt aus juristischer Sicht (Wildgans 2019) Zuckerbrot oder Peitsche? den Konstanzer Vorstoß zugunsten einer Zweitverwertungsmandatierung und plädiert insgesamt für eine Open-Access-Öffnung der juristischen Fachwissenschaft. Die hier vertretene These, dass mit der Erstpublikation das Veröffentlichungsrecht erschöpft ist, wird von den Juristen in Konstanz nicht berücksichtigt oder nicht geteilt. Ebenso wird die These offensichtlich nicht geteilt, dass die Öffentlichkeit einen Anspruch darauf hat, zu dem öffentlich finanziertem Wissen freien Zugang zu haben. Die Juristen in Konstanz sehen Wissenschaftsfreiheit wie auch Publikationsfreiheit nur als individuelles Recht. Das OLG München hat offenbar auf solche Argumente gar nicht reagiert, sondern lediglich die Kompetenz des Landes bzw. einer Universität für das Urheberrecht (Bundesrecht) bezweifelt.

426 (Höpfner/Amschewitz 2019) Die Zweitveröffentlichungspflicht im Spannungsfeld von OpenAccess-Kultur und Urheberrecht. 
sehene Zweitveröffentlichungspflicht zugunsten von Open Access auseinander. Die Universität war damit als bislang einzige Hochschule in Deutschland der Aufforderung des Landesgesetzgebers in Baden-Württemberg gefolgt. Höpfner war an der Klage als Professor in Konstanz beteiligt, ist aber jetzt nicht mehr dort tätig, also in dem noch offenen Verfahren der Klagebearbeitung nicht mehr involviert. Mit der Klage bzw. der jetzigen, die Klage unterstützenden Arbeit werden derart grundlegende Probleme angesprochen, so dass darauf etwas ausführlicher eingegangen werden soll.

Die Autoren machen unions- und verfassungsrechtliche Bedenken gegen diese Regelung geltend, aber auch den Verstoß gegen den Drei-Stufen-Test. Gegen das Unionsrecht würde verstoßen, weil das im Bund beschlossene Zweitverwertungsrecht zwar nicht gegen Unionsrecht verstoße, da dieses im Urhebervertragsrecht geregelt worden sei, wofür die EU keine Vorgaben gibt, dass aber eine Zweitveröffentlichungsverpflichtung als Eingriff in die Rechte der Urheber sehr wohl eine neue Schrankenregelung sei, die aber nach InfoSoc 2011 mit dem als abgeschlossenen geltenden Kanon von Schrankenregelungen nicht erlaubt sei. Das ist aber wohl ein Scheinargument, da eine Satzungsregelung einer Universität keine Urheberrechtsschranke entstehen lassen kann. Noch nicht abschließend beantwortet ist dadurch allerdings die vom Münchener OLG aufgeworfene Frage, ob die Regelung in Konstanz ein unzulässiger Eingriff in die Urheberrechtskompetenz des Bundes sei. Bezüglich der Verfassungsbedenken konzentrieren sich die Autoren weniger auf Wissenschaftsfreiheit als auf von ihnen gesehene Verstöße gegen die Eigentumsgarantie in Art. 14 Abs. 1. Hier vertreten die Verfasser eine extrem individualistische Sicht auf Eigentum und marginalisieren die Bedeutung von Art. 14 Abs. 2, der auf die Sozialbindung, hier verstanden als das öffentliche Interesse an der freien Nutzung des publizierten Wissens, abhebt. Sie sehen das vom BVerfG als Bedingung für Eingriffe in das Eigentum eingeforderte ,gesteigerte öffentliche Interesse“ ${ }^{427}$ nicht als gegeben an. Besonders wehren sich die Verfasser dagegen, dass die Zweitveröffentlichungspflicht für alle an der Universität Konstanz tätigen Wissenschaftler gelten soll, also auch für die Rechte an Werken, die aus der Grundfinanzierung entstanden sind. Sie beharren darauf - entgegen den oben erwähnten Interpretationen des Bundesrats und vielen Beiträgen aus der Fachwissenschaft, vor allem in den Kommentaren (s. oben) -, dass „nach dem eindeutigen Willen des Gesetzgebers“ das Zweitverwertungsrecht allein für Werke gelten soll, die aus der institutionell geförderten außeruniversitären Forschungseinrichtung“ oder durch „die öffentliche Drittmittelförderung“ entstanden seien. Offen ist hier, wieweit das Verständnis von „Gesetzgeber“ reichen soll.

427 Entsprechend BVerfGE 31, 229 (243) =NJW 1971, 2163. 
Der Bundestag, nicht die Bundesregierung hat das Gesetz verabschiedet. Aus dem Gesetz sind diese Einschränkungen nicht zu erkennen (gefordert ist nur eine mindestens zu $50 \%$ erfolgte öffentliche Finanzierung). Die Einschränkungen finden sich nur in der Begründung des Gesetzes durch die Bundesregierung. Sind diese dann zusammen mit dem Gesetzestext sozusagen der Wille des Gesetzgebers? Das mag gängige Auslegungspraxis der Gerichte sein, hat aber z. B. den Bundesrat nicht daran gehindert, eine verfassungskonforme Auslegung des Gesetzes anzumahnen. Es wäre zu begrüßen, wenn das BVerfG sich die Sache zu eigen und mit kreativer zeitgemäßer und undogmatischer Auslegung bestehender Gesetze und früherer Entscheidungen den Weg zu einer sanften Regelung des Zweitverwertungsrechts als auch institutionelles Recht freimachen würde - aus Gründen des „gesteigerten öffentlichen Interesses“.

\subsubsection{Fazit zum Zweitverwertungsrecht}

Das Zweitverwertungsgesetz ist für die Akteure in der Wissenschaft nur ein kleiner Fortschritt in Richtung Informationsautonomie der Urheber. Zu viele Einschränkungen, zu viele Zugeständnisse an Partikularinteressen einiger Verlage. Das Gesetz erfüllt nicht den angestrebten Zweck. Es ist zwar schwierig zu ermitteln, in welchem Ausmaß das Zweitverwertungsrecht überhaupt seitdem genutzt worden ist, aber alle Anzeichen aus den verschiedenen Bibliotheken deuten darauf hin, dass $\S 38$ Abs. 4 so gut wie gar nicht in Anspruch genommen wird. ${ }^{428}$ Wissenschaftler haben offensichtlich wenig Interesse daran, ihre Werke über Repositorien ein zweites Mal in einer zumal nicht zitierfähigen Version zu publizieren. Ebenso wird das von der Politik angestrebte Ziel der Beförderung von Open Access bzw. die rechtliche Absicherung des grünen Wegs von Open Access nicht erreicht - jedenfalls nicht durch $\S 38$ Abs. 4. Erst recht ist das Gesetz kein Fortschritt zugunsten des Gemeinwohlinteresses an freier Zugänglichkeit zu Wissen. Insgesamt eine zu späte, zu eingeschränkte und dann eher überflüssige Reform.

Manches läuft im Urheberrecht ganz anders als erwartet. So kann selbst ein nur gut gemeintes, aber doch schlechtes, unzureichendes Gesetz positive Folgen

\footnotetext{
428 Die Partei DIE LINKE hatte über Drucksache 19/704 vom 8.2.2018 eine kleine Anfrage zur Open-Access-Strategie des BMBF in den Bundestag eingebracht - https://bit.ly/2qSX53v. Unter Frage 16 wollte die LINKE wissen, „inwieweit das in $\S 38$ Absatz 4 des Urheberrechtsgesetzes eingeführte Zweitveröffentlichungsrecht zur Förderung von Open Access beigetragen hat“. In der Antwort heißt es nur: „Die Bundesregierung wird die Nutzung dieser Bestimmung im Rahmen ihrer Urheberrechtspolitik und Open-Access-Strategie auch in Zukunft beobachten“ - https: //bit.ly/2SteOmT.
} 
haben - ganz im Sinne der Als-ob-Philosophie Vaihingers. Auch Steinhauer sieht das Zweitverwertungsrecht als ein sehr unzulängliches an, so dass „die praktische Bedeutung des neuen Rechts ... daher eher gering [sein dürfte], seine Wirkung für mehr Open Access in der Wissenschaft aber groß”. So könne, nach Steinhauer, das

Zweitverwertungsrecht gerade in seiner unvollkommenen Ausgestaltung Verlage und Wissenschaft stimulieren, tragfähige und faire Geschäftsmodelle für die sofortige Publikation von Forschungsergebnissen im Netz gemeinsam zu entwickeln. ${ }^{429}$

Dem ist zuzustimmen. Tatsächlich wurde auch schon bei der abschließenden Diskussion über das Zweitverwertungsrecht im Bundestag deutlich, dass in Zukunft eher die Erstpublikation nach dem Open Access-(Gold)Paradigma im Vordergrund stehen sollte. Diese müsse „Teil der öffentlichen Forschungsförderung werden“: „Die vorliegende Gesetzesänderung sei insoweit Bestandteil eines übergreifenden Konzepts.“430

\subsection{Leistungsschutzrecht für Presseverleger}

Das Leistungsschutzrecht für Presseverleger - versprochen im Koalitionsvertrag 2009, beschlossen 2013, wegen Verletzung der Notationspflicht bei der EU vom EuGH 2019 für ungültig erklärt und auf Grund der verpflichtenden Vorgabe von EU-DMS-RL 2019 voraussichtlich 2020 wiedereingeführt - ist ein besonders krasses Beispiel für die Bestätigung der in 9 angeführten Thesen 2 und 3:

Den ökonomischen Interessen der Informationswirtschaft wird im Urheberrecht stärker Rechnung getragen als den Interessen der Allgemeinheit und der Akteure in Bildung und Wissenschaft.

Die politischen Instanzen unterstützen überwiegend eine die kommerzielle Verwertung sichernde Marktsicht auf Wissen und Information.

Mit Informationswirtschaft sind hier die Verlage im Presse-/Medienbereich angesprochen - nicht die Wirtschaft insgesamt. Die Wirtschaft, z. B. BDI, hatte sich überwiegend gegen dieses Leistungsschutzrecht ausgesprochen, ebenso viele Organisationen aus der Zivilgesellschaft und der Netz-Community, aber auch zahlreiche Bundespolitiker, nicht nur aus der Opposition (vor allem durch die LINKE). ${ }^{431}$ Aus der Sicht von Bildung und Wissenschaft besteht die Sorge, dass neue Leistungs-

429 (Steinhauer 2013) Mehr Open Access oder bloßer Placebo?

430 Bundestag Drucksache 17/14217 vom 26.6.2013 - https://bit.ly/2TVmeVj.

431 Das gesamte Verfahren, das pro und contra zum Leistungsschutzrecht wird ausführlich dokumentiert in (Brinkmann 2018) Verlagspolitik in der Zeitungskrise; vgl. auch (Koroch 2016). 
schutzrechte für Presseverlage die freizügige Nutzung von Medieninformation in Forschung und in der Ausbildung stark behindern könnten.

Vertreten wurden die Interessen für das Leistungsschutzrecht vor allem vom: Bundesverband Deutscher Zeitungsverleger e. V. (BDZV) und vom VDZ: Verband Deutscher Zeitschriftenverleger e. V. (VDZ). Deren intensives Lobbying fand besonders Gehör bei der Bundeskanzlerin, die sich persönlich zugunsten dieses Schutzrechts einsetzte, z. B. über eine Rede vor dem Zeitungskongress des BDZV im September 2011.432 Das Leistungsschutzrecht sollte, so die Erwartung der Presseverleger, bestätigen, dass Verlage das ausschließliche Recht haben, Artikel aus ihren Produkten zu gewerblichen Zwecken im Internet anzuzeigen und öffentlich zugänglich zu machen. ${ }^{433}$ Damit sollte die Praxis der Suchmaschinen und Nachrichtendiensten („Nachrichtenaggregatoren oder Medienbeobachtungsdiensten“434) abgewehrt werden, deren Geschäftsmodell darauf beruhe, „für die eigene Wertschöpfung auch auf die verlegerische Leistung zuzugreifen.“435 Diese Praxis sollte lizenzpflichtig werden, es sei denn diese Anbieter verwenden nur „einzelne Wörter oder kleinste Textausschnitte“ für ihre Anzeige. Betroffen davon seien, so die Bundesregierung (vgl. FN 435), nicht

andere Nutzer, wie z.B. Blogger, Unternehmen der sonstigen gewerblichen Wirtschaft, Verbände, Rechtsanwaltskanzleien oder private bzw. ehrenamtliche Nutzer. Die vorgeschlagene Regelung bedeutet damit keine Änderung der Nutzungsmöglichkeiten anderer Nutzer und für Verbraucher. Ihre Rechte und Interessen werden durch das vorgeschlagene Leistungsschutzrecht für Presseverleger nicht berührt.

Das Leistungsschutzrecht des Presseverlegers; ebenso auch der entsprechende Artikel in der Wikipedia - https://bit.ly/2UsB5J8; besonders kritisch aus der Zivilgesellschaft die Website IGEL. Initiative gegen ein Leistungsschutzrecht - https://leistungsschutzrecht.info/; aus der Web-Welt netzpolitik.org -https://bit.ly/2SdvmFW.

432 Angela Merkel 2011 Rede vor dem Zeitungskongress des BDZV: „Verlegerische Leistungen kosten Zeit und Geld. Deswegen kann ich auch gut verstehen, dass ein Leistungsschutzrecht für Verleger gefordert wird. Deshalb arbeitet die Bundesregierung derzeit an einem Gesetzentwurf, der das Urheberrecht weiter an die Anforderungen einer modernen Informationsgesellschaft anpassen soll. Wir haben es nicht vergessen; es wird vorangetrieben. Wir streben eine ausgewogene Regelung an, die den berechtigten Interessen aller Beteiligten Rechnung trägt.“ Vgl. dazu netethics. Gott, Merkel, nun noch ein Leistungsschutzrecht für Presseverleger - wie kann man die Netzwelt nur so missverstehen! - https://bit.ly/2SkX2HM.

433 Entsprechend § 87f Abs. 1 Satz 1: „Der Hersteller eines Presseerzeugnisses (Presseverleger) hat das ausschließliche Recht, das Presseerzeugnis oder Teile hiervon zu gewerblichen Zwecken öffentlich zugänglich zu machen, es sei denn, es handelt sich um einzelne Wörter oder kleinste Textausschnitte.“

434 So in EG 54 für Art. 11 der EU SDM-RL 2019 genannt.

435 Begründung der Bundesregierung (Drucksache 17/11470 vom 14,1,2912) - https://bit.ly/ 2GQ8gyr. 
Tatsächlich hat sich dieses in Deutschland eingeführte Leistungsschutzrecht für Presseverleger - so die überwiegende Einschätzung der Fachwelt außerhalb der Presselobby - als offensichtlicher Fehlschlag herausgestellt. Nicht zuletzt haben sich weder die Einnahmen der Verlage dadurch vergrößert, noch konnten die Journalisten als Autoren davon Nutzen ziehen. Auch waren kaum Bemühungen der Verlagswirtschaft zu erkennen, durch eigene Maßnahmen, die Anzeigepraxis z. B. der Suchmaschinen zu behindern oder sogar auszuschließen. ${ }^{436}$

Die deutsche Regelung des Leistungsschutzrechts von 2013 ist durch die Intervention des EuGH wegen Verletzung der Notationspflicht inzwischen hinfällig geworden (s. oben). Ein neues Tor für das Leistungsschutzrecht eröffnete sich aber 2019. Die EU hatte über DSM-RL Art. 15 (Schutz von Presseveröffentlichungen im Hinblick auf die Online-Nutzung) eine Verpflichtung der Mitgliedsländer festgeschrieben (,legen Bestimmungen fest“), nationale Leistungsschutzrechte in ihre nationale Gesetzgebung aufzunehmen. Entsprechend sah sich die Bundesregierung Mitte Januar 2020 in einem ersten Diskussionsvorschlag zur Umsetzung von DSM-RL in der Pflicht, das frühere „formale Defizit zeitnah zu korrigieren“ (https://bit.ly/39rcaLT). Das ist jetzt mit den $\S \S 87 \mathrm{f}-87 \mathrm{k}$ deutlich ausführlicher als in dem Versuch von 2013 ausgefallen. Die Bundesregierung wollte es mit dieser speziellen Umsetzung dieses nationalen Schutzrechts besonders eilig haben. Anders als die anderen Vorschläge zur Umsetzung, die erst zum 7.6.2021 in Kraft treten sollen, soll das Leistungsschutzrecht so schnell wie möglich erneut im Gesetz verankert werden. In $\S 87 \mathrm{~g}$ ist jetzt zur Verdeutlich die Formulierung der EU-Vorgabe aufgenommen: „das Setzen von Hyperlinks auf eine Presseveröffentlichung“ bleibt von dem Leistungsschutzrecht ausgeklammert. Weiter versucht die Bundesregierung in $\S 87 \mathrm{~g}$, der Einschränkung des neuen Rechts „Einzelne Wörter oder sehr kurze Auszüge“ den Charakter von unbestimmten Rechtsbegriffen dadurch zu nehmen, dass „einzeln“, „kurze“ spezifiziert wird, z. B. die Anzeige der Überschrift eines Textes sei erlaubt, ebenso das Zeigen eines kleinformatige Vorschaubild mit einer Auflösung von bis zu 128 mal 128 Pixeln und das Abspielen einer Tonfolge, Bildfolge oder Bild- und Tonfolge mit einer Dauer von bis zu drei Sekunden. Das ist, wie vieler solcher Versuch seit den Reformen ab 2003, durchaus satireverdächtig. ${ }^{437}$

436 Die Möglichkeit der Verlage, z. B. das Anzeigen von Presseinformation durch einfache technische Maßnahmen zu verhindern - z. B. durch Aufnahmen des Meta-Tag noindex in den Header des HTML-Codes der auszuschließenden Website mit der Konsequenz, dass Web-Scrawler blockiert werden - wird wohl deshalb kaum genutzt, weil die Presseverlage daran interessiert sind, die Sichtbarkeit ihrer Produkte über die Referenzen der Suchmaschinen weiter zu sichern (vgl. Information von Google Deutschland 2020 dazu - https://bit.ly/2SJ5XTs).

437 Vgl. Golem.de Leistungsschutzrecht: Memes sollen nur noch 128 mal 128 Pixel groß sein. 16.1.2010 - https://bit.ly/2VjUn3Z. 
Fazit. Leistungsschutzregelungen für Presseverlage in der jetzigen Form sind nicht zielführend - weder für Produzenten (Autoren und Verleger) noch für Nutzer. Sie könnten allenfalls dann sinnvoll sein, wenn a) sie lediglich auf von den Anbietern neu angebotenen Leistungen (informationelle Mehrwertprodukte und Metainformationsdienste) angewendet, b) durch das Leistungsschutzrecht bestehende Schrankenregelungen des Urheberrechts nicht ausgehebelt, c) die Rechte der produzierenden Urheber (hier in erster Linie der freien Journalisten) nicht beeinträchtigt und d) der freie Informationsfluss und die Orientierung der Nutzer im Internet nicht behindert werden. 